\title{
Review
}

\section{Animal Model for Glucocorticoid Induced Osteoporosis: A Systematic Review from 2011 to 2021}

\author{
Andy Xavier 1,2, Hechmi Toumi 1,2,3 (D) and Eric Lespessailles 1,2,3,*(D) \\ 1 EA 4708 I3MTO Laboratory, Orleans University, 45067 Orleans, France; andy.xavier@univ-orleans.fr (A.X.); \\ hechmi.toumi@univ-orleans.fr (H.T.) \\ 2 Translational Medicine Research Platform, PRIMMO, Regional Hospital of Orleans, 45007 Orleans, France \\ 3 Department Rheumatology, Regional Hospital of Orleans, 14 Avenue de L'Hopital, 45007 Orleans, France \\ * Correspondence: eric.lespessailles@chr-orleans.fr
}

check for updates

Citation: Xavier, A.; Toumi, H.; Lespessailles, E. Animal Model for Glucocorticoid Induced Osteoporosis: A Systematic Review from 2011 to 2021. Int. J. Mol. Sci. 2022, 23, 377. https://doi.org/10.3390/ ijms23010377

Academic Editor: Paola Marcolongo

Received: 7 December 2021

Accepted: 27 December 2021

Published: 29 December 2021

Publisher's Note: MDPI stays neutral with regard to jurisdictional claims in published maps and institutional affiliations.

Copyright: (C) 2021 by the authors. Licensee MDPI, Basel, Switzerland. This article is an open access article distributed under the terms and conditions of the Creative Commons Attribution (CC BY) license (https:// creativecommons.org/licenses/by/ $4.0 /)$.

\begin{abstract}
Clinical and experimental data have shown that prolonged exposure to GCs leads to bone loss and increases fracture risk. Special attention has been given to existing emerging drugs that can prevent and treat glucocorticoid-induced osteoporosis GIOP. However, there is no consensus about the most relevant animal model treatments on GIOP. In this systematic review, we aimed to examine animal models of GIOP centering on study design, drug dose, timing and size of the experimental groups, allocation concealment, and outcome measures. The present review was written according to the PRISMA 2020 statement. Literature searches were performed in the PubMed electronic database via Mesh with the publication date set between April, 2011, and February 2021. A total of 284 full-text articles were screened and 53 were analyzed. The most common animal species used to model GIOP were rats $(66 \%)$ and mice (32\%). In mice studies, males (58\%) were preferred and genetically modified animals accounted for $28 \%$. Our work calls for a standardization of the establishment of the GIOP animal model with better precision for model selection. A described reporting design, conduction, and selection of outcome measures are recommended.
\end{abstract}

Keywords: glucocorticoid induced osteoporosis; bone; animal models; bone loss; therapy; methylprednisolone; dexamethasone

\section{Introduction}

Despite their side effects, glucocorticoids (GCs) continue to be prescribed in many diseases because of their immunomodulatory capacities. However, therapy with long-term GCs leads to deleterious effects due to their systemic impact on the metabolism including cardiovascular, endocrine, dermatologic, muscular, and skeletal effects including bone fragility and aseptic osteonecrosis [1].

Glucocorticoid induced osteoporosis (GIOP) is the leading cause of secondary osteoporosis. GC intake is associated with large morbidity and increased mortality [2]. In patients with chronic corticosteroid therapy, the annual incidence rate of vertebral fractures was 3.2\% (95\% CI: $1.8-5)$ and 5.1\% (95\% CI: 2.8-8.2) in patients initiating treatment [3]. However, an increased risk of fragility fracture may be observed within the first three months of treatment $[3,4]$. Fragility fractures are the most common serious adverse events related to GIOP. Vertebral fractures, most often asymptomatic, may occur soon after exposure to GCs when bone mineral density (BMD) is rapidly decreasing [5]. Vertebral fractures are particularly associated with GIOP, although the risk of hip fractures is also increased [2,4].

Bone loss preferentially affects trabecular bone rather than cortical bone [6]. GCs can cause localized alterations in bone microarchitecture, resulting in micro-lesions that decrease bone strength. These localized alterations of microarchitecture have been shown to be correlated with GC intake [7]. These unique effects explain why GC exposure is associated with an increased risk of fracture at higher BMD values than in postmenopausal osteoporosis. The decrease in bone strength associated with GIOP seems to be rapidly 
reversible, as clinical observations showed that the prevalence of fractures decreased from the third month at the end of treatment $[5,8]$.

GCs alter the formation/resorption balance leading preferentially inhibition of bone formation $[4,7]$. During the first few months of treatment with GCs, the loss of bone density is greatest because GCs not only inhibit bone formation, but also accelerate bone resorption [6,9]. This transient increase in bone resorption has been shown to be in part related to treatment with GCs but also to the underlying inflammatory disease and it has been clearly demonstrated that inflammation promotes osteoclastic differentiation [10,11].

To further explore GIOP, it is of utmost importance to use animal models that show similarity to human pathophysiology, in order to carry out efficient preclinical studies and to test new compounds. Currently, the drugs used to treat GIOP are most often those used for postmenopausal osteoporosis (bisphosphonates, SERMs, and parathyroid hormone derivatives) [12]. However, these treatments mainly affect bone metabolism and do not correct other side effects of GIOP such as muscle wasting. These drugs are considered as active comparators in animal studies aiming at the development of new molecules. However, for preclinical studies, it remains difficult to find a suitable animal model that mimics human skeletal development. The use of large human-like animals such as non-human primates have been preferred due to their similarity concerning reproductive, anatomical, and physiological characteristics [13]. However, the use of these large animals such as dogs, pigs, and sheep remains limited due to ethical considerations and the difficulties associated with their maintenance and cost [13-15]. Small laboratory animals such as mice, rabbits, guinea pigs, and rats seem to meet these considerations and have already been used as animal models in postmenopausal osteoporosis research [16,17]. However, due to differences in rodent skeletal metabolism, their bone metabolism differs, which may limit translatability to human skeletal metabolism and constitutes a distinct challenge.

Given the number of animal models proposed, it is difficult to synthesize these studies to obtain a coherent understanding of the pathophysiology and select a reference animal model to test new therapies. Thus, herein, the aim of the present review is to provide a detailed overview of animal models of glucocorticoid-induced bone loss and explore how these models could be useful for preclinical and translational research on GIOP. We will also assess the quality of animal models by focusing on study design, drug dose, timing and size of the experimental groups, allocation concealment, and outcome measures.

\section{Materials and Methods}

\subsection{Protocol and Registration}

This systematic review was conducted and reported according to the PRISMA 2020 guidelines [18].

Our literature review was registered in the "Center for Reviews and Dissemination" PROSPERO; Registration number: CRD42021259669

\subsection{Eligibility Criteria}

The PICOS [18] (Population, Intervention, Comparator/Control Outcome, and Study design) concept was used to develop a search strategy.

Research has focused on animal studies of bone loss with glucocorticoid pharmacological treatment. In our review, we were interested in learning which animal models were the most relevant, robust, and reproducible. In addition, we focused on papers that measure, on one hand, the bone loss with either (dual-energy X-ray absorptiometry (DXA) or microtomography $(\mu \mathrm{CT})$, and on the other hand, biomechanical properties to characterize the GIOP bone phenotype.

\subsection{Information Sources}

The PubMed database was searched between January and April 2021. The last search was performed on 15 April 2021. 


\subsection{Search Strategy}

The studies were selected from a search strategy developed with an expert librarian on the PubMed database using the following keywords:

(osteoporo * OR "Osteoporosis" [Mesh] OR "bone loss" OR "glucocorticoid induced osteoporosis" OR "osteopenia" OR"Cancellous Bone" [Mesh] OR "Cortical Bone" [Mesh]) AND ("Glucocorticoids" [Mesh] OR "Methylprednisolone" [Mesh] OR "Dexamethasone" [Mesh]) AND ("models, animal" [Mesh] OR "Murinae" [Mesh] OR "Rabbits" [Mesh] OR "Sheep" [Mesh] NOT “Humans" [Mesh]) Filters: from 2011-2021

\subsection{Selection Process}

The inclusion criteria were as follows:

Experimental studies in animal models of glucocorticoid-induced osteoporosis or osteopenia in which the effects of glucocorticoid on biochemical markers, on bone tissues from the femur, the tibia or vertebrae were assessed through biochemical, biomechanical, histological, and imaging techniques. Studies were published in English and in internationally peer-reviewed journals between 2011 and 2021.

We excluded avian and zebra fish models as well as cellular models. Exclusion criteria also included studies related to osteonecrosis of the mandible or of the femoral head. We also excluded animal studies in which animals were also ovariectomized in order to limit our analysis and interpretation on GIOP mode. Ovariectomy in animals is a well-established model of bone loss in the literature for postmenopausal osteoporosis research $[16,19,20]$.

\subsection{Data Collection Process}

Data were extracted into a template established before starting the searches and then verified by double reading.

GIOP was defined as any intervention study where animals were treated with GCs and resulted in alteration of calcified bone tissue.

Several variables for which data was collected were defined: animals that were treated with GCs compared to a placebo control and had at least one medical imaging (DXA, $\mu C T$ ) and either histomorphometry or biomechanical studies. Two investigators (AX and EL) independently assessed all the studies and consensus was reached through discussion with a third investigator (HT).

\subsection{Data Items}

Information was extracted from each of the articles according to PICOS on the following criteria: (1) characteristics of the animal models (species, strain, sex, age, weight, number of animals used); (2) type of intervention (glucocorticoids used, dose, frequency, duration, administration; versus placebo or versus another GCs; or versus another drug used for the treatment of osteoporosis); and (3) type of outcome measure (imaging techniques (DXA or $\mu \mathrm{CT}$ ) and histomorphometry or biomechanical test (three point bending, micro-indentation, load test)).

\subsection{Study Risk of Bias Assessment}

This systematic review is exploratory in nature and aims to highlight the qualities and limitations of the species used for research in the GIOP. No risk of bias assessment was carried out. However, we assessed the methodological quality of the studies by referring to the work of Schulz et al. [21], particularly on the randomization of animal groups and statistical analysis.

\subsection{Effect Measures}

The studies should report DXA measurements expressed as BMC or BMD according to international recommendations. Micro-scanner or histomorphometry measurements should show microarchitecture parameters such as BV/TV, BS/BV, Tb.Th, Tb.Sp, Tb.N, 
as defined by Bouxsein and by Parfitt [22,23]. Finally, the strength tests must include at least one extrinsic parameter measurement [24,25].

\subsection{Synthesis Methods}

A study was eligible for our search if it included at least one animal model (described above) in which a glucocorticoid drug intervention (dexamethasone, methylprednisolone, prednisone, prednisolone, and cortisone) was performed compared to a placebo control group. The study also had to include imaging measurements of bone tissue (DXA or $\mu \mathrm{Ct}$ or histomorphometry) or a strength test.

The selected studies were analyzed and classified in tables. If any of the information was missing or not clearly identified, the information was inferred from the article understanding or labeled not available (NA). For example, the number of subjects could be deduced from the results of the statistical tests.

\section{Results}

\subsection{Study Selection}

The search strategy identified 284 papers in the PubMed database. Based on title or abstract screening, 245 studies were excluded since they did not meet the eligibility criteria. The subsequent full-text assessment resulted in 39 records that were found eligible for the comprehensive review. Fourteen additional articles from our own personal records were included as they fell within the eligibility criteria of our work but did not emerge from the search strategy. Finally, 53 articles were considered (Figure 1).

\section{Literature search from 2011 to Avril 2021}

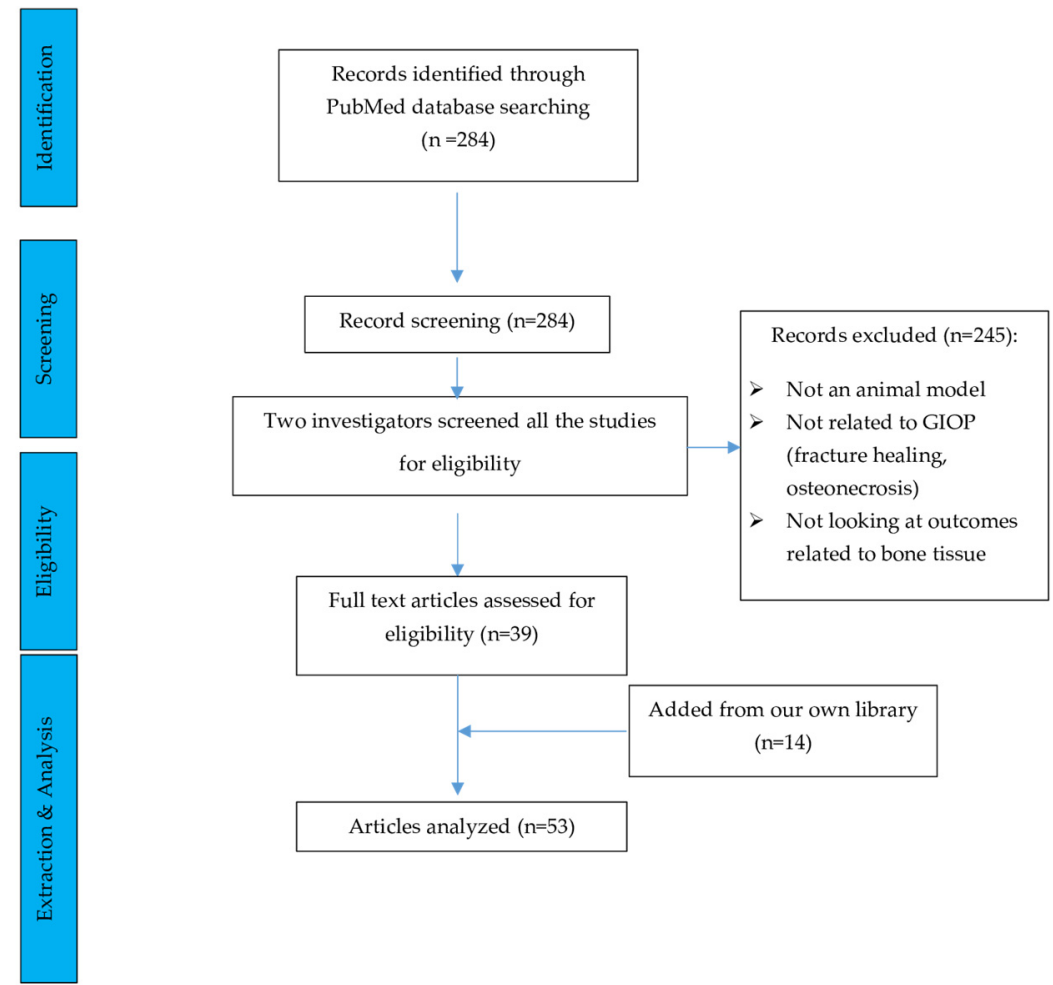

Figure 1. Flowchart illustrating the study selection process.

\subsection{Animal Characteristics}

The majority of the studies selected and analyzed used rats $(n=34)$ as experimental models with a predominance for the Sprague Dawley strain. Studies using mice represented a third of the studies $(n=18)$ with a predominance of the C57BL/ 6 strain and its numerous knockout derivatives. One study used male C57BL/ 6 mice and male Sprague Dawley rats, 
which was counted twice in Table 1. Finally, one study in rabbit and one in sheep were included (Table 1).

Regarding the sex of the animals, in rats of all strains, there was no marked predominance. In mice, it appeared that males constituted the majority (55\%). Finally, the few studies in rabbit and sheep did not allow us to establish a trend.

In Sprague Dawley rats (male and female), the age range was from eight weeks to six months with rats averaging three and a half months old. The age range varied from a few days (neonatal) to six months in the other strains.

In C57BL/ 6 mice, the age range was eight weeks to four months; in the other strains, the age range was seven weeks to four months. Age of rabbit (one study [26]) and sheep (one study [27]) is indicated in Table 2.

Table 1. Number of articles by species, strain, and sex in the GIOP model. One paper [28] included both mice and rats with different protocols.

\begin{tabular}{|c|c|c|c|c|c|c|c|c|}
\hline & \multicolumn{3}{|c|}{ Rat (34 Protocols) } & \multicolumn{3}{|c|}{ Mice (18 Protocols) } & \multirow{2}{*}{$\begin{array}{c}\begin{array}{c}\text { Rabbit } \\
\text { (1 Protocol) }\end{array} \\
\begin{array}{c}\text { New } \\
\text { Zealand } \\
\text { White }\end{array} \\
\end{array}$} & \multirow{2}{*}{$\begin{array}{c}\begin{array}{c}\text { Sheep } \\
\text { (1 Protocol) }\end{array} \\
\text { Merino }\end{array}$} \\
\hline & $\begin{array}{l}\text { Sprague } \\
\text { Dawley }\end{array}$ & Wistar & $\begin{array}{l}\text { Albinos } \\
\text { /LEW CrlCrlj }\end{array}$ & C57BL/6 & $\begin{array}{l}\text { C57BL/6 with } \\
\text { Sprague Dawley }\end{array}$ & $\begin{array}{l}\text { Other } \\
\text { Strain }\end{array}$ & & \\
\hline Male & 11 & 3 & 1 & 3 & 1 & 6 & 1 & \\
\hline Female & 14 & 2 & 1 & 6 & & 0 & & 1 \\
\hline Male/Female & 1 & 1 & & & & 1 & & \\
\hline Sex Not available & & & & & & 1 & & \\
\hline $\begin{array}{c}\text { Total } \\
\text { articles }\end{array}$ & 26 & 6 & 2 & 9 & 1 & 8 & 1 & 1 \\
\hline
\end{tabular}

Table 2. Main characteristics of the proposed experimental protocol to induce GIOP in rabbit and sheep. $\mathrm{M}=$ Male, $\mathrm{F}$ = Female DEX = Dexamethasone, $\mathrm{IM}=$ Intramuscular injection, $\mathrm{SC}$ = Subcutaneous injection.

\begin{tabular}{|c|c|c|c|c|c|c|c|c|c|}
\hline References & Species & Strain & Sex & Age & Weight & Molecule Used & Administration & Dosage & Duration \\
\hline $\begin{array}{c}2014 \mathrm{Z} \\
\text { Yongtao [26] }\end{array}$ & Rabbit & $\begin{array}{c}\text { New Zealand } \\
\text { White }\end{array}$ & $\mathrm{M}$ & $32 \mathrm{w}$ & $3.2 \pm 0.45 \mathrm{~kg}$ & DEX & IM & $\begin{array}{c}3 \mathrm{mg} / \mathrm{kg} \text { twice } \\
\text { per week }\end{array}$ & $12 \mathrm{~W}$ \\
\hline $\begin{array}{c}2011 \mathrm{M} \\
\text { Ding [27] }\end{array}$ & Sheep & Merino & $\mathrm{F}$ & $4-6 Y$ & $55 \pm 10 \mathrm{~kg}$ & Prednisolone & SC & $\begin{array}{c}0.60 \mathrm{mg} / \mathrm{kg} \\
5 \text { times } \\
\text { per weeks }\end{array}$ & $7 \mathrm{M}$ \\
\hline
\end{tabular}

\subsection{Induction of GIOP}

The GCs used in murine models of GIOP are dexamethasone (DEX) (49\%), prednisone $(22 \%)$, and methylprednisolone (MP) (14\%) in rats, and in mice, prednisolone (47\%), MP (24\%), and DEX (12\%) (Figure 2).

The route of administration in rats was mainly subcutaneous (SC) (45\%), intramuscular (IM) $(22 \%)$, and Per. Os (P.O) (22\%). The intraperitoneal (IP) and intravenous (IV) routes were less used (Tables 3-5. In mice, the most commonly used route was SC through pellets inserted surgically (pellet in subcutaneous: PSC) (61\%). Other routes of administration were less used (P.O: 16\%, SC: 16\%) (Tables 6 and 7). In rabbit and sheep, the routes of administration were IM and SC (Table 2).

In rats, DEX was administered at the lowest dose of $0.1 \mathrm{mg} / \mathrm{kg}$ daily for 60 days [29] and at the highest dose of $25 \mathrm{mg} / \mathrm{kg}$ twice per week for six weeks [30]. Prednisone was administered at the lowest dose of $1.5 \mathrm{mg} / \mathrm{kg}$ per day for 90 days [31] and at the highest dose of $6 \mathrm{mg} / \mathrm{kg}$ per day for 90 weeks [31]. In mice, prednisolone was administered at the lowest dose of $0.8 \mathrm{mg} / \mathrm{kg}$ per day for three weeks [32] and at the highest dose of $4 \mathrm{mg} / \mathrm{kg}$ per day for three weeks [32]. In rabbits and sheep, DEX was administered at a dose of $3 \mathrm{mg} / \mathrm{kg}$ twice weekly for 12 weeks [26] and prednisolone at a dose of $0.6 \mathrm{mg} / \mathrm{kg}$ five times weekly for seven months [27], respectively. 


\subsection{Quality Assessment}

Only 38 studies reported random assignment of animals to groups. Of these studies, $23 \%$ reported the method of randomization, either by animal weight or by initial BMD measurement. Only eight studies reported had blinded assessment of outcomes.

Although the majority of studies presented a quality protocol, it should be noted that $43 \%$ did not report the weight of the animals, $7 \%$ the age, $3 \%$ the number of subjects used in the protocol, and $2 \%$ did not specify the sex of the animals. A minority of studies did not clearly indicate the dose and duration of the treatment used. Protocols that used the P.O route did not always specify the mode of administration.

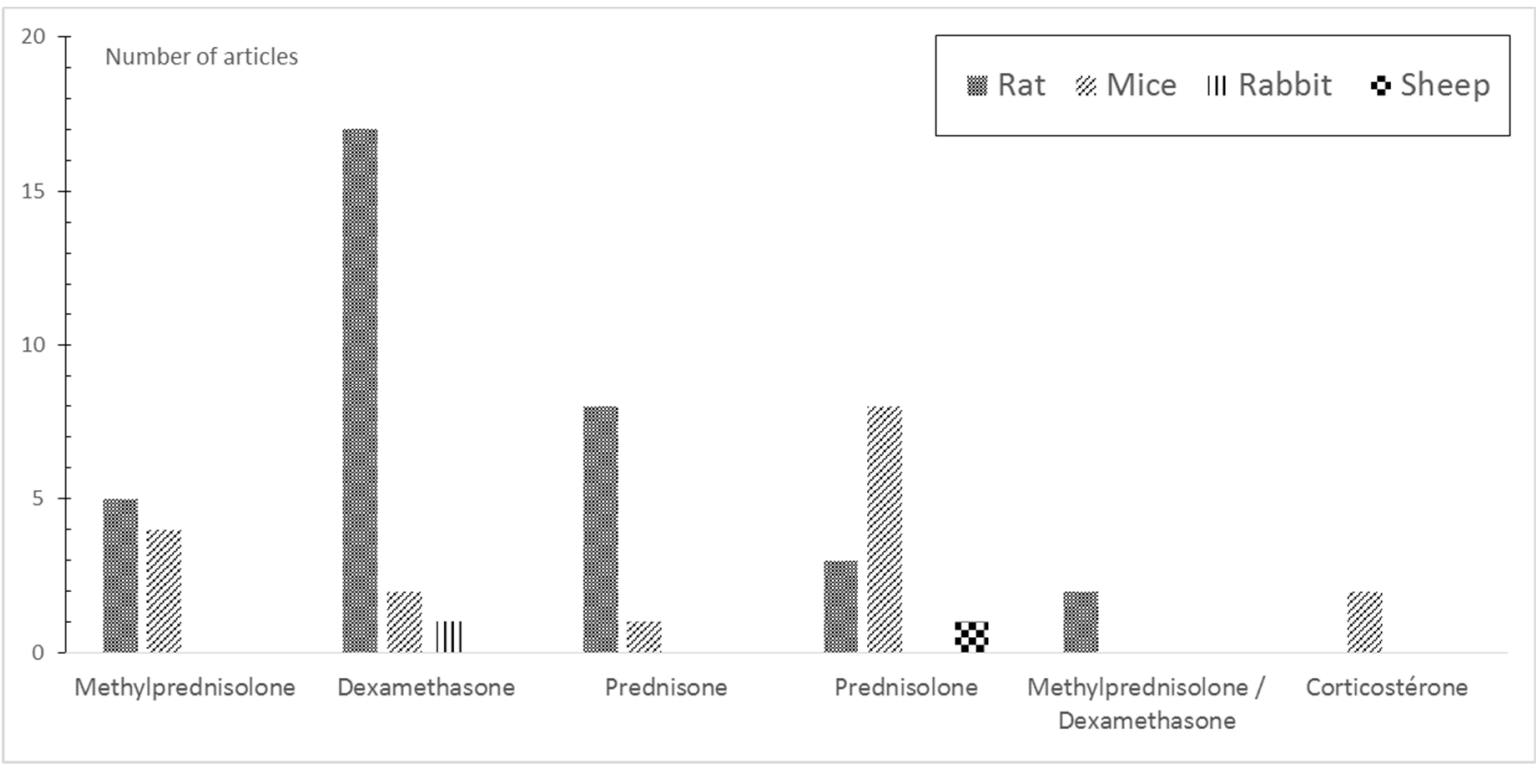

Figure 2. Distribution of glucocorticoid use by species and number of articles.

Table 3. Main characteristics of the proposed experimental protocol to induce GIOP in female Sprague Dawley. DEX = Dexamethasone, MP = Methylprednisolone, IM = Intramuscular injection, $\mathrm{SC}=$ Subcutaneous injection, IV = Intravenous injection, IP = Intraperitoneal injection, P.O = Per. Os, NA $=$ Not Available.

\begin{tabular}{|c|c|c|c|c|c|c|}
\hline References & Age & Weight & $\begin{array}{c}\text { Molecule } \\
\text { Used }\end{array}$ & $\begin{array}{c}\text { Route of } \\
\text { Administration }\end{array}$ & Dosage & Duration \\
\hline 2019 Y Xu [33] & $8 \mathrm{~W}$ & $250 \pm 10 \mathrm{~g}$ & DEX & $\mathrm{IM}$ & $2.5 \mathrm{mg} / \mathrm{kg}$ twice per week & $2 \mathrm{M}$ \\
\hline 2019 J Zhao [34] & $3 \mathrm{M}$ & $280 \pm 14 \mathrm{~g}$ & $\mathrm{MP}$ & SC & $13 \mathrm{mg} / \mathrm{kg} 5$ days per week & $9 \mathrm{~W}$ \\
\hline 2018 Y Yang [35] & $4 \mathrm{M}$ & $225 \pm 25 \mathrm{~g}$ & Prednisone & P.O & $5 \mathrm{mg} / \mathrm{kg}$ daily & $14 \mathrm{~W}$ \\
\hline 2017 H Ren [36] & $3 \mathrm{M}$ & NA & DEX & SC & $0.6 \mathrm{mg} / \mathrm{kg}$ every 3 days & $3 \mathrm{M}$ \\
\hline 2017 M Zhou [37] & $6 \mathrm{M}$ & $200 \pm 20 \mathrm{~g}$ & Prednisone & P.O & $6 \mathrm{mg} / \mathrm{kg}$ daily & $21 \mathrm{~W}$ \\
\hline 2017 G Chen [38] & $4-5 \mathrm{M}$ & $250-275 \mathrm{~g}$ & Prednisone & P.O & $5 \mathrm{mg} / \mathrm{kg}$ daily & $90 \mathrm{D}$ \\
\hline 2016 Z Chen [29] & NA & NA & DEX & SC & $0.1 \mathrm{mg} / \mathrm{kg}$ daily & $60 \mathrm{D}$ \\
\hline 2016 Y Yang [39] & $4 \mathrm{M}$ & $200-250 \mathrm{~g}$ & Prednisone & P.O & $5 \mathrm{mg} / \mathrm{kg}$ daily & $14 \mathrm{~W}$ \\
\hline 2016 G Shen [40] & $3 \mathrm{M}$ & NA & DEX & SC & $0.6 \mathrm{mg} / \mathrm{kg}$ twice per week & $3 \mathrm{M}$ \\
\hline 2015 H Ren [41] & $3 \mathrm{M}$ & $212 \pm 30 \mathrm{~g}$ & $\begin{array}{l}\text { DEX } \\
\text { MP }\end{array}$ & SC & $\begin{array}{c}0.6 \mathrm{mg} / \mathrm{kg} \text { twice per week } \\
1 \mathrm{mg} / \mathrm{kg} \text { daily }\end{array}$ & $12 \mathrm{~W}$ \\
\hline 2013 M Khan [42] & NA & $180 \pm 20 \mathrm{~g}$ & $\begin{array}{l}\text { DEX } \\
\text { MP }\end{array}$ & $\begin{array}{l}\text { IP } \\
\text { SC }\end{array}$ & $\begin{array}{l}200 \mu \mathrm{\mu g} / \mathrm{kg} 5 \text { days per week } \\
5 \mathrm{mg} / \mathrm{kg} 5 \text { days per week }\end{array}$ & $4 \mathrm{~W}$ \\
\hline 2017 G Pizzino [43] & $5 \mathrm{M}$ & $250-275 \mathrm{~g}$ & MP & SC & $30 \mathrm{mg} / \mathrm{kg}$ & $60 \mathrm{D}$ \\
\hline 2016 Y Jiang [44] & $3 \mathrm{M}$ & $210 \pm 20 \mathrm{~g}$ & DEX & $\mathrm{IM}$ & $\begin{array}{l}2.5 \mathrm{mg} / \mathrm{kg} \text { twice per week } \\
0.6 \mathrm{mg} / \mathrm{kg} \text { twice per week }\end{array}$ & $12 \mathrm{~W}$ \\
\hline 2016 D Liang [45] & $4 \mathrm{M}$ & NA & DEX & SC & $\begin{array}{c}\text { (prevention) } \\
0.6 \mathrm{mg} / \mathrm{kg} \text { daily (treatment) }\end{array}$ & $3 \mathrm{M}$ \\
\hline 2015 Y Liu [46] & $12 \mathrm{~W}$ & $263.5 \pm 12 \mathrm{~g}$ & DEX & IV & $2 \mathrm{mg} / \mathrm{kg}$ twice per week & $12 \mathrm{~W}$ \\
\hline
\end{tabular}


Table 4. Main characteristics of the proposed experimental protocol to induce GIOP in male Sprague Dawley. $\mathrm{DEX}=$ Dexamethasone, $\mathrm{MP}=$ Methylprednisolone, $\mathrm{IM}=$ Intramuscular injection, SC $=$ Subcutaneous injection, $\mathrm{P} . \mathrm{O}=$ Per. Os, NA = Not Available, ${ }^{\dagger}$ Study using mice and Sprague Dawley rats.

\begin{tabular}{|c|c|c|c|c|c|c|}
\hline References & Age & Weight & $\begin{array}{l}\text { Molecule } \\
\text { Used }\end{array}$ & Administration & Dosage & Duration \\
\hline $2021 \mathrm{Y} \mathrm{Mo} \mathrm{[28]}^{\dagger}$ & $4 \mathrm{M}$ & NA & Prednisone & P.O & $5 \mathrm{mg} / \mathrm{kg}$ daily & $16 \mathrm{~W}$ \\
\hline 2020 S Pal [47] & NA & $260 \pm 20 \mathrm{~g}$ & $\mathrm{MP}$ & SC & $5 \mathrm{mg} / \mathrm{kg}$ daily & $4 \mathrm{~W}$ \\
\hline 2019 L. Yang [48] & $8 \mathrm{~W}$ & $220 \pm 10 \mathrm{~g}$ & DEX & IM & $1 \mathrm{mg} / \mathrm{kg}$ twice per week & $3 \mathrm{M}$ \\
\hline 2014 M Feng [49] & $6 \mathrm{M}$ & $220-240 \mathrm{~g}$ & DEX & SC & $0.1 \mathrm{mg} / \mathrm{kg}$ daily & $5 \mathrm{~W}$ \\
\hline 2013 Z Ren [50] & $5 \mathrm{M}$ & $390 \mathrm{~g}$ & DEX & SC & $0.1 \mathrm{mg} / \mathrm{kg}$ daily & $5 \mathrm{~W}$ \\
\hline 2013 F-S Wang [51] & $5 \mathrm{M}$ & NA & $\mathrm{MP}$ & SC & $5 \mathrm{mg} / \mathrm{kg}$ daily & $1-2$ or $4 \mathrm{~W}$ \\
\hline 2011 F-S Wang [52] & $5 \mathrm{M}$ & NA & DEX & SC & $0.1 \mathrm{mg} / \mathrm{kg}$ daily & $1-2$ or $5 \mathrm{~W}$ \\
\hline 2012 L Cui [53] & $6 \mathrm{M}$ & $390 \pm 25 \mathrm{~g}$ & Prednisone & P.O & $3.5 \mathrm{mg} / \mathrm{kg}$ daily & $12 \mathrm{~W}$ \\
\hline 2017 Y Yang [54] & $12 \mathrm{~W}$ & $200 \pm 20 \mathrm{~g}$ & DEX & $\mathrm{IM}$ & $\begin{array}{c}1 \mathrm{mg} / \mathrm{kg} \text { twice per week } \\
1.5 \mathrm{mg} / \mathrm{kg} \text { daily }\end{array}$ & $8 \mathrm{~W}$ \\
\hline 2014 S Lin [31] & $3 \mathrm{M}$ & $300 \mathrm{~g}$ & Prednisone & P.O & $\begin{array}{l}3.0 \mathrm{mg} / \mathrm{kg} \text { daily } \\
6.0 \mathrm{mg} / \mathrm{kg} \text { daily }\end{array}$ & $90 \mathrm{D}$ \\
\hline 2012 J-Y Ko [55] & $4 \mathrm{M}$ & NA & DEX & SC & $0.1 \mathrm{mg} / \mathrm{kg}$ daily & $1-2$ or $5 \mathrm{~W}$ \\
\hline 2017 M Zhou [37] & $6 \mathrm{M}$ & $220 \pm 20 \mathrm{~g}$ & Prednisone & P.O & $6 \mathrm{mg} / \mathrm{kg}$ daily & $21 \mathrm{~W}$ \\
\hline
\end{tabular}

Table 5. Main characteristics of the proposed experimental protocol to induce GIOP in male and female rats (excluding Sprague Dawley). M = Male, F = Female, DEX = Dexamethasone, MP = Methylprednisolone, $\mathrm{IM}=$ Intramuscular injection, $\mathrm{SC}=$ Subcutaneous injection, $\mathrm{PSC}=$ Pellet in Subcutaneous, NA = Not Available.

\begin{tabular}{|c|c|c|c|c|c|c|c|c|}
\hline References & Strain & Sex & Age & Weight & $\begin{array}{l}\text { Molecule } \\
\text { Used }\end{array}$ & Administration & Dosage & Duration \\
\hline 2020 Y Yang [56] & Wistar & $\mathrm{F}$ & $6 \mathrm{~W}$ & $180 \pm 20 \mathrm{~g}$ & DEX & IM & $\begin{array}{c}2.5 \mathrm{mg} / \mathrm{kg} \text { twice } \\
\text { per week }\end{array}$ & $7 \mathrm{~W}$ \\
\hline 2020 D Sato [57] & $\begin{array}{c}\mathrm{LEW} \\
\mathrm{CrlCrlj}\end{array}$ & $\mathrm{F}$ & $5 \mathrm{~W}$ & $125 \mathrm{~g}$ & Prednisolone & PSC & $0.42 \mathrm{mg}$ daily & $6 \mathrm{~W}$ \\
\hline 2019 T Hou [58] & Albinos & M & Neo-natal & $5-10 \mathrm{~g}$ & DEX & NA & $0.1 \mathrm{mg} / \mathrm{kg}$ & $6 \mathrm{~W}$ \\
\hline $\begin{array}{l}2017 \text { L.M.F. } \\
\text { Lucinda [59] }\end{array}$ & Wistar & $\mathrm{F}$ & $50 \mathrm{D}$ & $100-150 \mathrm{~g}$ & DEX & $\mathrm{IM}$ & $7 \mathrm{mg} / \mathrm{kg}$ once per week & $5 \mathrm{~W}$ \\
\hline 2016 N Han [30] & Wistar & $\mathrm{M} / \mathrm{F}$ & $3 \mathrm{M}$ & $283 \pm 42 \mathrm{~g}$ & DEX & IM & $25 \mathrm{mg} / \mathrm{kg}$ twice per week & $6 \mathrm{~W}$ \\
\hline 2015 Z Achiou [60] & Wistar & $\mathrm{M}$ & $19 \mathrm{~W}$ & $450 \mathrm{~g}$ & MP & SC & $5 \mathrm{mg} / \mathrm{kg} 5$ days per week & $9 \mathrm{~W}$ \\
\hline 2013 K Pichler [61] & Wistar & M & $12 \mathrm{~W}$ & $240 \pm 20 \mathrm{~g}$ & Prednisolone & SC & $7 \mathrm{mg} / \mathrm{kg}$ daily & $4 \mathrm{~W}$ \\
\hline 2011 M Saito [62] & Wistar & M & $6 \mathrm{M}$ & $330 \mathrm{~g}$ & Prednisolone & IM & $\begin{array}{c}10 \mathrm{mg} / \mathrm{kg} 5 \text { days } \\
\text { per week }\end{array}$ & $4 \mathrm{~W}$ \\
\hline
\end{tabular}

Table 6. Main characteristics of the proposed experimental protocol to induce GIOP in male and female C57BL $/ 6$ and $\mathrm{KO}$ derivate. $\mathrm{M}=$ Male, $\mathrm{F}=$ Female, $\mathrm{DEX}=$ Dexamethasone, $\mathrm{P} . \mathrm{O}=\mathrm{Per}$. Os, PSC $=$ Pellet in Subcutaneous, SC $=$ Subcutaneous injection, NA $=$ Not Available ${ }^{\dagger}{ }^{\dagger}$ Study using mice and rat Sprague Dawley.

\begin{tabular}{|c|c|c|c|c|c|c|c|}
\hline References & Sex & Age & Weight & Molecule Used & Administration & Dosage & Duration \\
\hline $2021 \mathrm{Y} \mathrm{Mo} \mathrm{[28]}^{\dagger}$ & $M$ & $8 \mathrm{~W}$ & NA & Prednisone & P.O & $2.1 \mathrm{mg} / \mathrm{kg}$ daily & $8 \mathrm{~W}$ \\
\hline 2017 A Y Sato [63] & $\mathrm{F}$ & $16 \mathrm{~W}$ & NA & Prednisolone & PSC & $2.1 \mathrm{mg} / \mathrm{kg}$ daily & 14 or $28 \mathrm{D}$ \\
\hline 2016 A Y Sato [64] & $\mathrm{F}$ & $4 \mathrm{M}$ & $25 \pm 6 \mathrm{~g}$ & Prednisolone & PSC & $\begin{array}{l}1.4 \mathrm{mg} / \mathrm{kg} \text { daily } \\
2.1 \mathrm{mg} / \mathrm{kg} \text { daily }\end{array}$ & $90 \mathrm{D}$ \\
\hline 2016 A Ersek [65] & $\mathrm{F}$ & $12 \mathrm{~W}$ & NA & Prednisolone & PSC & $2.5 \mathrm{mg}$ & $60 \mathrm{D}$ \\
\hline 2019 Q Geng [66] & M & $12 \mathrm{~W}$ & NA & DEX & SC & $\begin{array}{c}1 \mathrm{mg} / \mathrm{kg} 5 \text { days } \\
\text { per week }\end{array}$ & $4 \mathrm{~W}$ \\
\hline 2019 L Mao [67] & $\mathrm{F}$ & $10 \mathrm{~W}$ & $20 \pm 2.0 \mathrm{~g}$ & DEX & SC & $\begin{array}{l}10 \mathrm{mg} / \mathrm{kg} \text { three } \\
\text { times per week }\end{array}$ & $90 \mathrm{D}$ \\
\hline 2019 CG Fenton [68] & M & $9 \mathrm{~W}$ & NA & Corticosterone & P.O & $\begin{array}{c}100 \mu \mathrm{g} / \mathrm{mL} \text { twice } \\
\text { per week }\end{array}$ & $4 \mathrm{~W}$ \\
\hline 2019 J D Schepper [69] & M & $15 \mathrm{~W}$ & NA & Prednisolone & PSC & $2.5 \mathrm{mg} / \mathrm{kg}$ daily & $60 \mathrm{D}$ \\
\hline 2018 C Ohlsson [70] & $\mathrm{F}$ & $12 \mathrm{~W}$ & NA & $\mathrm{MP}$ & PSC & $7.6 \mathrm{mg} / \mathrm{kg}$ daily & $4 \mathrm{~W}$ \\
\hline 2018 I Bergström [71] & $\mathrm{F}$ & $3 \mathrm{M}$ & NA & Prednisolone & PSC & $11 \mathrm{mg} / \mathrm{kg}$ daily & $11 \mathrm{D}$ \\
\hline
\end{tabular}


Table 7. Main characteristics of the proposed experimental protocol to induce GIOP in mice (excepted C57BL $/ 6$ and their KO derivatives). $\mathrm{M}=$ Male, $\mathrm{F}=$ Female, DEX = Dexamethasone, $\mathrm{MP}=$ Methylprednisolone, $\mathrm{P} . \mathrm{O}=$ Per. Os, $\mathrm{SC}=$ Subcutaneous injection, PSC = Pellet in Subcutaneous, $\mathrm{IP}=$ Intraperitoneal injection, NA = Not Available.

\begin{tabular}{|c|c|c|c|c|c|c|c|c|}
\hline References & Strain & Sex & Age & Weight & $\begin{array}{l}\text { Molecule } \\
\text { Used }\end{array}$ & Administration & Dosage & Duration \\
\hline $\begin{array}{c}2021 \text { A M } \\
\text { Dubrovsky [72] }\end{array}$ & $\mathrm{BALB} / \mathrm{cJ}$ & M & $9 \mathrm{~W}$ & NA & MP & PSC & $2.5 \mathrm{mg}$ for 21 day/pellet & $\begin{array}{l}60 \text { or } \\
120 \mathrm{D}\end{array}$ \\
\hline $\begin{array}{c}2019 \text { S } \\
\text { Adhikary [73] }\end{array}$ & $\mathrm{BALB} / \mathrm{C}$ & M & $8 \mathrm{~W}$ & $22-25 \mathrm{~g}$ & MP & $\mathrm{SC}$ & $10 \mathrm{mg} / \mathrm{kg}$ & $10 \mathrm{~W}$ \\
\hline $\begin{array}{c}2018 \text { I } \\
\text { Alam [74] }\end{array}$ & $\begin{array}{c}\text { Col2.3- } \\
\text { hWNT16TG }\end{array}$ & $\mathrm{M} / \mathrm{F}$ & $16 \mathrm{~W}$ & NA & Prednisolone & PSC & $2.1 \mathrm{mg} / \mathrm{kg}$ daily & $28 \mathrm{D}$ \\
\hline $\begin{array}{c}2017 \mathrm{G} \\
\text { Mohan [75] }\end{array}$ & Swiss Webster & M & $4 \mathrm{M}$ & NA & Prednisolone & PSC & $2.8 \mathrm{mg} / \mathrm{kg}$ daily & $28-56 \mathrm{D}$ \\
\hline $\begin{array}{l}2016 \text { F-S } \\
\text { Wang [76] }\end{array}$ & $\begin{array}{c}129 \mathrm{~S} \\
\text { Npytm1RPA/J }\end{array}$ & M & $16 \mathrm{~W}$ & NA & MP & IP & $5 \mathrm{mg} / \mathrm{kg}$ daily & $4 \mathrm{~W}$ \\
\hline $\begin{array}{l}2015 \mathrm{~W} \\
\text { Yao [32] }\end{array}$ & $\begin{array}{l}\text { dsRed-LC3 } \\
\text { report }\end{array}$ & M & $2 \mathrm{M}$ & NA & Prednisolone & PSC & $0.8-2.8-4 \mathrm{mg} / \mathrm{kg}$ daily & $3 \mathrm{~W}$ \\
\hline $\begin{array}{c}2011 \mathrm{M} \\
\text { Marenzana [77] }\end{array}$ & $\mathrm{BALB} / \mathrm{c}$ & NA & $7 \mathrm{~W}$ & $22.5 \mathrm{~g}$ & DEX & P.O & $3 \mathrm{mg} / \mathrm{kg}$ daily & $\begin{array}{c}6 \mathrm{~W} \text { or } \\
9 \mathrm{~W}\end{array}$ \\
\hline $\begin{array}{c}2011 \mathrm{H} \\
\text { Henneicke [78] }\end{array}$ & Col2.3-11ßHSD2 & M & $8 \mathrm{~W}$ & NA & Corticosterone & PSC & $\begin{array}{c}1.5 \mathrm{mg} / \mathrm{kg} \text { implanted at } \\
0-1-14 \text { or } 21 \text { days }\end{array}$ & $28 \mathrm{D}$ \\
\hline
\end{tabular}

\subsection{Techniques to Measure GIOP}

The number of articles using DXA $\mu \mathrm{CT}$ histomorphometry and mechanical test by animal species and strain is reported in Table 8 . In rats, $85 \%$ of the studies used mechanical testing, $82 \%$ DXA, $52 \% \mu \mathrm{CT}$, and $47 \%$ histomorphometry. In mice, $85 \%$ of the studies used $\mu \mathrm{CT}, 72 \%$ mechanical testing, 50\% histomorphometry, and $44 \%$ DXA.

Table 8. Number of studies by species and strain using bone measurement techniques.

\begin{tabular}{ccccccc}
\hline & \multicolumn{2}{c}{ Rat } & \multicolumn{2}{c}{ Mice } & Rabbit & Sheep \\
\hline & $\begin{array}{c}\text { Sprague } \\
\text { Dawley }\end{array}$ & $\begin{array}{c}\text { Other } \\
\text { Strain }\end{array}$ & C57BL/6 & $\begin{array}{c}\text { Other } \\
\text { Strain }\end{array}$ & $\begin{array}{c}\text { New Zealand } \\
\text { White }\end{array}$ & Merino \\
\hline DXA & 20 & 8 & 5 & 3 & 1 & 1 \\
$\mu C T$ & 17 & 1 & 8 & 7 & & 1 \\
Mechanical Test & 23 & 6 & 6 & 7 & 1 & 1 \\
Histomorphometry & 13 & 3 & 6 & 3 & 1 & \\
\hline
\end{tabular}

\subsection{Bone Loss, Micro Architecture Alteration, and Decrease in Bone Strength Induced by GCs}

We listed all significant results of bone loss microarchitecture alteration and decrease in bone strength induced by GCs assessed by DXA, $\mu \mathrm{CT}$, histomorphometry, and biomechanical testing in Table 9. A total of $87 \%$ of the studies reported alterations in microarchitecture observed either by $\mu \mathrm{CT}$ or histomorphometry. Of these studies, $63 \%$ reported trabecular bone analysis, $28 \%$ trabecular and cortical bone analyses, and $8 \%$ cortical analysis. 
Table 9. Results of bone quality assessments reported by the studies. The DXA parameters are BMD (bone mineral density) and BMC (bone mineral content). The $\mu \mathrm{CT}$ parameters are BV/TV (bone volume/tissue volume), BS/BV (bone surface/bone volume), Tb.N (trabecular number), and Tb.Th (trabecular thickness), Tb.Sp (trabecular separation), SMI (structure-model index). The used static histomorphometry parameters are: Oc.N/BS (osteoclast number/bone surface), Ob.N/BS (osteoblast number/bone surface), Oc/S/BS (osteoclast surface/bone surface), Ob.S/BS (osteoblast surface/bone surface), ES/BS (eroded surface/bone surface), Oc.Pm/B.Pm (osteoclast perimeter/bone perimeter), Ob.Pm/B.Pm (osteoblast perimeter/bone perimeter), OS/BS (osteoid surface/bone surface). The dynamic histomorphometry parameters are: MS/BS (mineralizing surface/bone surface), MAR (mineral apposition rate), BFR/BS (bone formation rate/bone surface).

\begin{tabular}{|c|c|c|c|}
\hline References & $\begin{array}{l}\text { Bone Loss Measuring } \\
\text { by DXA }\end{array}$ & $\begin{array}{c}\text { Alterations of the Microarchitecture } \\
\text { Observed by } \mu \mathrm{CT} \text { or Static/Dynamic } \\
\text { Histomorphometry }\end{array}$ & $\begin{array}{c}\text { Loss in Bone Strength Parameters } \\
\text { Observed by Biomechanical } \\
\text { Testing }\end{array}$ \\
\hline 2019 Y Xu [33] & Yes at femur (BMC BMD) & $\begin{array}{c}\text { Yes at femur (BV/TV, Tb.Th, Tb.Sp, Tb.N) } \\
\text { by } \mu \mathrm{CT}\end{array}$ & $\begin{array}{l}\text { Yes at femur (maximum stress (MPa), } \\
\text { maximum load }(\mathrm{N}) \text {, elasticity modulus } \\
\left(\mathrm{N} / \mathrm{mm}^{2}\right)\end{array}$ \\
\hline 2019 J Zhao [34] & $\begin{array}{l}\text { Yes at femur and L5 } \\
\text { vertebra (BMD) }\end{array}$ & $\begin{array}{c}\text { Yes at proximal tibia (BV/TV, Tb.Th, Tb.Sp, } \\
\text { Tb.N, \%L.Pm, MAR, BFR/BV, BFR/TV, } \\
\text { BFR/BS, Oc.N/BS, Oc.Pm/BS) } \\
\text { by histomorphometry }\end{array}$ & NA \\
\hline 2018 Y Yang [35] & NA & $\begin{array}{l}\text { Yes at proximal femur (Tb.Ar, Tb.Th, } \\
\text { BV/TV, Tb.Sp, SMI, DA) by } \mu \mathrm{CT}\end{array}$ & $\begin{array}{l}\text { Yes at femur (elastic load }(\mathrm{N}) \text {, bending } \\
\text { energy }(\mathrm{N} \times \mathrm{mm})\end{array}$ \\
\hline 2017 H Ren [36] & $\begin{array}{l}\text { Yes at L1-L5 vertebrae (BMD, } \\
\text { BMC, AREA) }\end{array}$ & $\begin{array}{l}\text { Yes at L2 vertebra (BS/TV, BV/TV, Tb.Th, } \\
\text { Tb.Sp, Tb.N, vBMD) by } \mu \mathrm{CT}\end{array}$ & $\begin{array}{l}\text { Yes at L2 vertebra (compressive strength } \\
(\mathrm{N}) \text {, compressive displacement }(\mathrm{mm}) \\
\text { energy absorption capacity }(\mathrm{J}))\end{array}$ \\
\hline 2017 M Zhou [37] & $\begin{array}{l}\text { Yes (BMD BMC at the L4 } \\
\text { vertebra, whole femur in } \\
\text { male; BMD proximal femur, } \\
\text { BMC distal femur in female) }\end{array}$ & $\begin{array}{c}\text { Yes (at the distal femur in female: BV/TV, } \\
\text { density, SMI, Tb.N, Tb.Th, Tb.Sp; at the } \\
\text { distal femur in male: BV/TV, density) } \\
\text { by } \mu \mathrm{CT}\end{array}$ & $\begin{array}{l}\text { Yes (at the femur in male elastic load }(\mathrm{N}) \text {, } \\
\text { stiffness }(\mathrm{N} / \mathrm{mm}) \text { at the femur in female: } \\
\text { elastic load }(\mathrm{N}) \text {; at the L5 vertebra in } \\
\text { female: elastic load }(\mathrm{N}) \text {, maximum load }(\mathrm{N}) \text {, } \\
\text { break load }(\mathrm{N}) \text {, stiffness }(\mathrm{N} / \mathrm{mm}))\end{array}$ \\
\hline 2017 G Chen [38] & Yes at femur (BMD, BMC) & $\begin{array}{c}\text { Yes at proximal tibia (Tb.Th, SMI by } \mu \mathrm{CtT} \text {, } \\
\text { Tb.Ar, Tb.N, Tb.Th, Tb.Sp } \\
\text { by histomorphometry) }\end{array}$ & $\begin{array}{c}\text { Yes at femur (maximum load }(\mathrm{N}) \text {, Breaking } \\
\text { load }(\mathrm{N}) \text {, Yield load }(\mathrm{N}) \text {, bending } \\
\text { energy }(\mathrm{mJ}))\end{array}$ \\
\hline 2016 Z Chen [29] & Yes at proximal femur (BMD) & NA & $\begin{array}{l}\text { Yes at femur (ultimate load (N), Stiffness } \\
\qquad(\mathrm{N} / \mathrm{mm}))\end{array}$ \\
\hline 2016 Y Yang [39] & NA & $\begin{array}{l}\text { Yes at proximal femur (BV/TV, Tb.Th, } \\
\text { Tb.Sp); at proximal metaphysis tibia: } \\
\text { \%L.Pm, BFR/TV) by histomorphometry }\end{array}$ & $\begin{array}{l}\text { Yes at femur (fracture load }(\mathrm{N}) \text {, Bending } \\
\text { energy } \mathrm{N} \times \mathrm{mm}) \text { ) }\end{array}$ \\
\hline 2016 G Shen [40] & $\begin{array}{l}\text { Yes at L1-L3 vertebrae (BMC, } \\
\text { BMD, AREA) }\end{array}$ & $\begin{array}{l}\text { Yes at L4 vertebra (BS/TV, BV/TV, Tb.Th, } \\
\text { Tb.Sp, Tb.N, vBMD, SMI) by } \mu \mathrm{CT}\end{array}$ & $\begin{array}{l}\text { Yes at L4 vertebra (compressive strength } \\
(\mathrm{N}) \text {, compressive stiffness } \\
(\mathrm{N} / \mathrm{mm}) \text {,compressive displacement }(\mathrm{mm}), \\
\text { energy absorption capacity }(\mathrm{N}))\end{array}$ \\
\hline 2015 H Ren [41] & $\begin{array}{l}\text { Yes at L1-L3 vertebrae (BMC, } \\
\text { BMD, AREA) }\end{array}$ & $\begin{array}{c}\text { Yes at L4 vertebra for DEX group and MP } \\
\text { group (BV/TV, BS/TV, SMI, Tb.Th, vBMD) } \\
\text { only DEX group (Conn.D, Tb.Sp, Tb.N) } \\
\text { by } \mu \mathrm{CT}\end{array}$ & $\begin{array}{l}\text { Yes at L4 vertebra for DEX group and MP } \\
\text { group (compressive strength(N)) }\end{array}$ \\
\hline 2013 MP Khan [42] & NA & $\begin{array}{l}\text { Yes at hypophysis/diaphysis femur and } \\
\text { tibia (vBMD, BV/TV, Conn.D, SMI, Tb.Th, } \\
\text { Tb.Sp, Tb.N, Porosity, DA) by } \mu \mathrm{CT} \text {; (Ct/th, } \\
\text { MAR, pBFR/BS) and by histomorphometry }\end{array}$ & $\begin{array}{l}\text { Yes at femur (ultimate load }(\mathrm{N}) \text {, Energy } \\
(\mathrm{mJ}) \text {, Stiffness }(\mathrm{N} / \mathrm{mm}))\end{array}$ \\
\hline 2017 G Pizzino [43] & Yes at femur (BMD) & $\begin{array}{c}\text { Yes at femur and vertebra (BV/TV, Tb.Th) } \\
\text { by } \mu \mathrm{CT}\end{array}$ & Yes at femur (maximum load $(\mathrm{N})$ ) \\
\hline 2016 Y Jiang [44] & $\begin{array}{l}\text { No statistical difference } \\
\text { reported at femur and whole } \\
\text { body (BMD, BMC) }\end{array}$ & $\begin{array}{l}\text { Yes at L4 vertebra (Tb.Ar, Tb.N, Tb.Sp) } \\
\text { by histomorphometry }\end{array}$ & NA \\
\hline 2016 D Liang [45] & $\begin{array}{l}\text { Yes at L1-L4 vertebrae (BMC, } \\
\text { BMD) }\end{array}$ & $\begin{array}{l}\text { Yes at L2 vertebra (BV/TV, SMI, Tb.N, } \\
\text { Tb.Th, Tb.Sp, vBMD) by } \mu C T\end{array}$ & $\begin{array}{l}\text { Yes at L2 vertebra (compressive strength } \\
(\mathrm{N}) \text {, compressive displacement }(\mathrm{mm}) \\
\text { energy absorption capacity }(\mathrm{J}))\end{array}$ \\
\hline
\end{tabular}


Table 9. Cont.

\begin{tabular}{|c|c|c|c|}
\hline References & $\begin{array}{l}\text { Bone Loss Measuring } \\
\text { by DXA }\end{array}$ & $\begin{array}{l}\text { Alterations of the Microarchitecture } \\
\text { Observed by } \mu \mathrm{CT} \text { or Static/Dynamic } \\
\text { Histomorphometry }\end{array}$ & $\begin{array}{l}\text { Loss in Bone Strength Parameters } \\
\text { Observed by Biomechanical } \\
\text { Testing }\end{array}$ \\
\hline 2015 Y Liu [46] & $\begin{array}{l}\text { Yes at femur (BMD) and no } \\
\text { statistical difference reported } \\
\text { at L5 vertebra }\end{array}$ & $\begin{array}{c}\text { Yes at proximal tibia by } \mu \mathrm{CT}(\mathrm{BV} / \mathrm{TV}, \\
\text { Tb.Th, Tb.N, Tb.Sp) and } \\
\text { by histomorphometry (BV/TV, Tb.N, Tb.Sp, } \\
\text { MS/BS, MAR, BFR/BS, BFR/BV, OcS, } \\
\text { OcS/BS) }\end{array}$ & $\begin{array}{l}\text { Yes at femur (energy }(\mathrm{J}) \text {, Bending stiffness } \\
\qquad(\mathrm{N} / \mathrm{mm}))\end{array}$ \\
\hline 2021 Y Mo [28] & NA & $\begin{array}{l}\text { Yes in rat at distal femur (vBMD) by } \mu \mathrm{CT} \text {; } \\
\text { at proximal tibia: \% } \% \text {.Ar, Tb.Wi, Oc.N, } \\
\text { \%Oc.Pm, \%Ob.Pm) by histomorphometry; } \\
\text { and in mice at distal femur (VBMD, Tb.Th) } \\
\text { and by } \mu \mathrm{CT}\end{array}$ & $\begin{array}{l}\text { Yes at femur in rat (maximum load }(\mathrm{N}) \text {, } \\
\text { fracture load }(\mathrm{N}) \text {, stiffness }(\mathrm{N})) \text { and in mice } \\
(\text { elastic load }(\mathrm{N}))\end{array}$ \\
\hline 2020 S Pal [47] & NA & $\begin{array}{c}\text { Yes at femur (vBMD, BV/TV, Tb.N, Tb.SP, } \\
\text { Tb.Th, SMI, BMD, Ct.Th, Periosteal } \\
\text { perimeter) at L5 vertebra (vBMD, BV/TV, } \\
\text { Tb.N, SMI) by } \mu \mathrm{CT}\end{array}$ & $\begin{array}{c}\text { Yes at femur (peak load }(\mathrm{N}) \text {, energy }(\mathrm{mJ}) \\
\text { stiffness }(\mathrm{N} / \mathrm{mm}))\end{array}$ \\
\hline 2019 L. Yang [48] & NA & $\begin{array}{l}\text { Yes at vertebra (BMD, TMD, Conn.D, Tb.Th, } \\
\text { Tb.Sp) at femur (BMD, TMD, Conn.D, } \\
\text { Tb.Th, Tb.Sp, Tb.N, BV /TV) by } \mu C T\end{array}$ & $\begin{array}{l}\text { Yes at femur (Bending load (N), Elastic } \\
\text { modulus (MPa)) }\end{array}$ \\
\hline 2014 M Feng [49] & Yes at femur (BMD) & $\begin{array}{c}\text { Yes at proximal femur (BFR/BF, N.Ot, } \\
\text { N.Ob, BV/TV, Tb.Th, N.OC/BS, Tb.Sp) } \\
\text { by histomorphometry }\end{array}$ & NA \\
\hline 2013 Z Ren [50] & Yes at femur (BMD) & $\begin{array}{c}\text { Yes at proximal femur (BV/TV, Tb.N, } \\
\text { Tb.Th, Tb.Sp) by } \mu \mathrm{CT}\end{array}$ & Yes at femur (Peak load $(\mathrm{N}))$ \\
\hline 2013 F-S Wang [51] & Yes at femur (BMD, BMC) & Yes at femur (BV/TV, Ct. Porosity) by $\mu \mathrm{CT}$ & Yes at femur $(\operatorname{Load}(\mathrm{N}))$ \\
\hline 2011 F-S Wang [52] & $\begin{array}{l}\text { Yes but bone site no } \\
\text { reported (BMD) }\end{array}$ & $\begin{array}{c}\text { Yes at proximal tibia (BMC) by } \mu \mathrm{CT} ; \\
\text { (BFR/BS, BV/TV, Ob surface, Oc surface) } \\
\text { and by histomorphometry }\end{array}$ & $\begin{array}{l}\text { Yes but bone site no reported (Peak load } \\
\qquad(\mathrm{N}))\end{array}$ \\
\hline 2012 L Cui [53] & $\begin{array}{l}\text { Yes at proximal and whole } \\
\text { femur (BMD), measured } \\
\text { by single photon }\end{array}$ & $\begin{array}{l}\text { Yes at proximal tibia (BV/TV, Tb.Wi, } \\
\text { ObS/BS, LGR, MAR, BFR/TV, Ec.MS/BS, } \\
\text { EC.MAR, Ec.BFR/BS) } \\
\text { by histomorphometry }\end{array}$ & $\begin{array}{l}\text { Yes at femur (maximum force }(\mathrm{N}) \text {, } \\
\text { maximum deflection }(\mathrm{mm}) \text { ) }\end{array}$ \\
\hline 2017 Y Yang [54] & Yes at whole femur (BMD) & $\begin{array}{l}\text { Yes at proximal metaphysis femur (Tb.Ar, } \\
\text { Tb.Th, Tb.Sp, Tb.N) by histomorphometry }\end{array}$ & $\begin{array}{c}\text { Yes at femur (maximal load }(\mathrm{kg}) \text {, ultimate } \\
\text { deflection }(\mathrm{mm}) \text { ) }\end{array}$ \\
\hline 2014 S Lin [31] & $\begin{array}{l}\text { Yes at whole femur (BMD } \\
\text { (Significant result at } 6 \\
\mathrm{mg} / \mathrm{kg} / \mathrm{d} \text { prednisolone } \\
\text { in femur)) }\end{array}$ & $\begin{array}{c}\text { Yes at proximal tibia (Tb.Th, MS/BS, MAR, } \\
\text { BFR/BS, BFR/BV) (significant result at } 6 \\
\text { mg/kg/d prednisolone in tibia); Ob.S/BS, } \\
\text { Oc.S/BS (significant result for doses below } \\
6 \text { mg/kg/d prednisolone in tibia); (Tb.Th, } \\
\text { Tb.N, Tb.Sp (significant result at } 3 \\
\text { mg/kg/d prednisolone in femur); (Ps } \\
\text { MAR, Ps.BFR/BS (significant result at } 3 \\
\text { mg/kg/d prednisolone in tibia } \\
\text { shaft)(Ct.Th, Ec.MS/BS, Ec.MAR, } \\
\text { Ec.BFR/BS (significant result at all dose)) } \\
\text { by histomorphometry }\end{array}$ & $\begin{array}{l}\text { Yes at femur and L5 vertebra (elastic load } \\
(\mathrm{N}) \text {, maximum load(N) fracture load }(\mathrm{N}) \\
\text { stiffness coefficient }(\mathrm{N} / \mathrm{mm}) \text { (Significant } \\
\text { result at } 6 \mathrm{mg} / \mathrm{kg} / \mathrm{d} \text { prednisolone in } \\
\text { femur)); maximum load }(\mathrm{N}), \text { Young's } \\
\text { modulus at L5 vertebra (MPa)) }\end{array}$ \\
\hline 2012 J-Y Ko [55] & $\begin{array}{l}\text { Yes but bone site no reported } \\
\text { (BMD, BMC) }\end{array}$ & $\begin{array}{c}\text { Yes (BMC) by } \mu \mathrm{CT} ;(\mathrm{BV} / \mathrm{TV}, \mathrm{Ob} . \mathrm{S} / \mathrm{BS}, \\
\text { Oc.S/BS, BFR/BS) and } \\
\text { by histomorphometry }\end{array}$ & Yes at tibia (peak load $(\mathrm{N})$ \\
\hline 2020 Y Yang [56] & $\begin{array}{l}\text { Yes at femur (BMD, BMC } \\
\text { (measured by single photon } \\
\text { bone mineral } \\
\text { density analyzer)) }\end{array}$ & $\begin{array}{l}\text { Yes at femur (trabecular area index) } \\
\text { by histomorphometry }\end{array}$ & NA \\
\hline 2020 D Sato [57] & Yes at distal femur (BMD) & $\begin{array}{c}\text { Yes at proximal tibia (Oc.Pm/B.Pm, ES/BS, } \\
\text { N.Oc/BS, OS/BS, Ob.S/BS, BFR/BS) } \\
\text { by histomorphometry }\end{array}$ & Yes at tibia (maximum stress $\left(\mathrm{N} / \mathrm{cm}^{3}\right)$ ) \\
\hline 2019 T Hou [58] & Yes at proximal tibia (BMD) & NA & $\begin{array}{l}\text { Yes at femur (peak load }(\mathrm{N}) \text {, ultimate } \\
\text { stiffness }(\mathrm{N} / \mathrm{mm}) \text { ) }\end{array}$ \\
\hline $\begin{array}{l}\text { 2017 L.M.F. } \\
\text { Lucinda [59] }\end{array}$ & Yes (BMD) at tibia & NA & $\begin{array}{l}\text { Yes at tibia (maximum load }(\mathrm{N}) \text {, bone } \\
\text { stiffness }(\mathrm{N} / \mathrm{m}) \text {, energy }(\mathrm{mJ})\end{array}$ \\
\hline
\end{tabular}


Table 9. Cont.

\begin{tabular}{|c|c|c|c|}
\hline References & $\begin{array}{l}\text { Bone Loss Measuring } \\
\text { by DXA }\end{array}$ & $\begin{array}{l}\text { Alterations of the Microarchitecture } \\
\text { Observed by } \mu \mathrm{CT} \text { or Static/Dynamic } \\
\text { Histomorphometry }\end{array}$ & $\begin{array}{l}\text { Loss in Bone Strength Parameters } \\
\text { Observed by Biomechanical } \\
\text { Testing }\end{array}$ \\
\hline 2016 N Han [30] & Yes (BMC, BMD) at femur & NA & $\begin{array}{l}\text { Yes at femur (Flexure strength }(\mathrm{Mpa}) \text {, } \\
\text { maximum bending force }(\mathrm{N}))\end{array}$ \\
\hline 2015 Z Achiou [60] & Yes (BMC, BMD) at femur & $\begin{array}{l}\text { Yes (BV/TV, Tb.N, Tb.Th, Tb.Pf, Ct.Ar, } \\
\text { Ct.Th) at femoral mid-diaphysis by } \mu \mathrm{CT}\end{array}$ & No statistical difference measured \\
\hline 2013 K Pichler [61] & $\begin{array}{l}\text { Yes (BMD) at whole body, } \\
\text { vertebra, and femur }\end{array}$ & $\begin{array}{l}\text { No statistical difference reported at femur } \\
\text { by histomorphometry }\end{array}$ & NA \\
\hline 2011 M Saito [62] & $\begin{array}{l}\text { No statistical difference } \\
\text { reported }\end{array}$ & NA & $\begin{array}{l}\text { Yes at femur (maximum load }(\mathrm{N}) \text {, energy } \\
(\mathrm{mJ}))\end{array}$ \\
\hline 2017 A Y Sato [63] & $\begin{array}{l}\text { Yes (BMD (at whole body at } \\
14 \text { days and whole body, } \\
\text { femur, L1-L6 vertebrae at } \\
28 \text { days) }\end{array}$ & $\begin{array}{l}\text { Yes (Tb.Th) at distal femur and proximal } \\
\text { tibia by } \mu \mathrm{CT} \text {; (BFR/BS, MAR, MS/BS) } \\
\text { proximal tibia (periosteal and endocortical) } \\
\text { and by histomorphometry }\end{array}$ & NA \\
\hline 2016 A Y Sato [64] & $\begin{array}{l}\text { Yes (BMD) at whole body and } \\
\text { L1-L6 vertebrae }\end{array}$ & $\begin{array}{l}\text { Yes (Tb.Th, BA/TA, total Ct.Th, dorsal } \\
\text { Ct.Th) by } \mu \text { CT at L6 vertebra; (MAR, } \\
\text { BFR/BS, N.Oc/BS, Oc.S/BS) and } \\
\text { by histomorphometry at L1-L3 vertebrae }\end{array}$ & $\begin{array}{l}\text { Yes at L6 vertebra (ultimate force }(\mathrm{N}) \text {, } \\
\text { energy to ultimate load }(\mathrm{mJ}) \text {, toughness } \\
\left.\qquad\left(\mathrm{mJ} / \mathrm{mm}^{3}\right)\right)\end{array}$ \\
\hline 2016 A Ersek [65] & NA & $\begin{array}{l}\text { Yes (BV/TV, Tb.N, Tb.Pf, SMI, Ct.Th) at } \\
\text { vertebra by } \mu \mathrm{CT} \text { (N.Oc/T.Ar, Oc.S/BS) at } \\
\text { vertebra and by histomorphometry }\end{array}$ & $\begin{array}{l}\text { Yes at femur (maximum load }(\mathrm{N}) \text {, elastic } \\
\text { modulus }(\mathrm{MPa}) \text { ) }\end{array}$ \\
\hline 2019 Q Geng [66] & $\begin{array}{l}\text { Yes }(\mathrm{BMC}) \text { at total body, } \\
\text { vertebrae, and femur }\end{array}$ & $\begin{array}{l}\text { Yes (BV/TV, Tb.N, Tb.Th, Ct.Th, Ct.V, SMI, } \\
\text { Tb.Sp) at femur by } \mu \text { CT; (BV/TV, T.Col, } \\
\text { N.Ob/BS, Ob.S/BS, MS/BS, MAR BFR/BS) } \\
\text { at distal femur and by histomorphometry }\end{array}$ & $\begin{array}{l}\text { Yes at femur (maximum load }(\mathrm{N}) \text {, energy } \\
\text { absorption }(\mathrm{N} \times \mathrm{mm}) \text {, stiffness }(\mathrm{N} / \mathrm{M}), \\
\text { ultimate displacement }(\mu \mathrm{m}), \text { yield } \\
\text { displacement }(\mu \mathrm{m}), \text { yield load }(\mathrm{N}))\end{array}$ \\
\hline 2019 L Mao [67] & Yes (BMD) at femur & NA & Yes at tibia (ultimate load, stiffness) \\
\hline $\begin{array}{l}2019 \text { CG Fenton } \\
\text { [68] }\end{array}$ & NA & $\begin{array}{l}\text { Yes (BV/TV, Tb.N, Tb.Th, Tb.Sp) at tibia } \\
\text { by } \mu \mathrm{CT} \text {; (N.Ob/B.Pm) at L3-4 vertebrae } \\
\text { by histomorphometry }\end{array}$ & NA \\
\hline $\begin{array}{l}2019 \text { J D Schepper } \\
\text { [69] }\end{array}$ & NA & $\begin{array}{c}\text { Yes (BV/TV/BW, Tb.Sp, Tb.Th, Tb.N, } \\
\text { BV/TV) at femur by } \mu \mathrm{CT}\end{array}$ & No statistical difference reported \\
\hline 2018 C Ohlsson [70] & Yes (BMC) at total body & $\begin{array}{l}\text { Yes }(\mathrm{BV} / \mathrm{TV}) \text { at femur by } \mu \mathrm{CT} ;(\mathrm{Tb} . \mathrm{Th}, \\
\text { MAR, Ct.Th, Endosteal circumference, } \\
\text { Ct.Po, BFR) at femur and } \\
\text { by histomorphometry }\end{array}$ & NA \\
\hline $\begin{array}{l}2018 \text { I Bergström } \\
\text { [71] }\end{array}$ & NA & $\begin{array}{l}\text { Yes (cBMC, Ps.Pm, Imoment of inertia, } \\
\text { moment of resistance, Ct.Ar, Ct.Th) at } \\
\text { proximal tibia by } \mu \mathrm{CT}\end{array}$ & No statistical difference reported \\
\hline $\begin{array}{c}2021 \text { A M } \\
\text { Dubrovsky [72] }\end{array}$ & NA & $\begin{array}{l}\text { Yes (Ct.Ar, Ct.Ar/Tt.Ar, CT.Th) at central } \\
\text { femur; (Tb.Th) at distal femur by } \mu \mathrm{CT}\end{array}$ & $\begin{array}{c}\text { Yes (Yield load }(\mathrm{N})) \text { at femur; (ultimate load } \\
(\mathrm{N}) \text {, Yield load }(\mathrm{N}) \text {, work to ultimate force } \\
(\mathrm{N} \mathrm{mm}) \text { for } 120 \text { days treatment at L6 } \\
\text { vertebra. }\end{array}$ \\
\hline $\begin{array}{l}2019 \text { S Adhikary } \\
\text { [73] }\end{array}$ & NA & $\begin{array}{c}\text { Yes (vBMD, BV/TV, Tb.N, Conn.Den, } \\
\text { Tb.Sp, SMI) at epiphysis femur; (CT.Ar, } \\
\text { T.Ar, T.Pm) at diaphysis femur; (vBMD, } \\
\text { Ct.Th, T.Ar, B.Ar, T.Pm, MMI) at disphysis } \\
\text { tibia by } \mu \mathrm{CT}\end{array}$ & $\begin{array}{l}\text { Yes at femur (stiffness }(\mathrm{N}) \text {, energy }(\mathrm{mJ}) \\
\text { power }(\mathrm{N}))\end{array}$ \\
\hline 2018 I Alam [74] & $\begin{array}{l}\text { Yes (aBMD, BMC (only in } \\
\text { female)) at femur }\end{array}$ & $\begin{array}{c}\text { Yes (only in Female) (Tb.N, Tb.Sp) at L5 } \\
\text { vertebra; BA/TA, Ct.Th, pMOI,) at femur } \\
\text { by } \mu \mathrm{CT}\end{array}$ & $\begin{array}{l}\text { Yes at femur (stiffness, ultimate force, } \\
\text { energy to ultimate force (only in female)) }\end{array}$ \\
\hline 2017 G Mohan [75] & NA & $\begin{array}{l}\text { Yes (BV/TV, Ct.BV (only treatment study)) } \\
\text { at distal and mid-shaft femur by } \mu \mathrm{CT}\end{array}$ & $\begin{array}{l}\text { Yes (maximum load (prevention ( } 28 \text { days) } \\
\text { and treatment ( } 56 \text { days) study)) at L6 } \\
\text { vertebra; (maximum load (only treatment } \\
\text { study) at femur }\end{array}$ \\
\hline 2016 F-S Wang [76] & Yes (BMC, BMC) at femur & $\begin{array}{l}\text { Yes (B.Ar/T.Ar, Tb.Th, Tb.N, Tb.Sp, Bv/TV, } \\
\text { BFR/BS, Ob.S, Oc.S) at femur } \\
\text { by histomorphometry }\end{array}$ & NA \\
\hline
\end{tabular}


Table 9. Cont.

\begin{tabular}{|c|c|c|c|}
\hline References & $\begin{array}{l}\text { Bone Loss Measuring } \\
\text { by DXA }\end{array}$ & $\begin{array}{c}\text { Alterations of the Microarchitecture } \\
\text { Observed by } \mu \mathrm{CT} \text { or Static/Dynamic } \\
\text { Histomorphometry }\end{array}$ & $\begin{array}{c}\text { Loss in Bone Strength Parameters } \\
\text { Observed by Biomechanical } \\
\text { Testing }\end{array}$ \\
\hline 2016 N Han [30] & Yes (BMC, BMD) at femur & NA & $\begin{array}{l}\text { Yes at femur (Flexure strength }(\mathrm{Mpa}) \text {, } \\
\text { maximum bending force }(\mathrm{N}))\end{array}$ \\
\hline 2015 W Yao [32] & NA & $\begin{array}{c}\text { Yes (BV/TV) by } \mu \mathrm{CT} \text {; (Tb.Th, Conn.D (at } 4 \\
\text { mg/kg/d prednisolone)) at L5 vertebra } \\
\text { by histomorphometry; (BMD, Ec-MS/BS, } \\
\text { Ps-BFR at } 2.8 \mathrm{mg} / \mathrm{kg} / \mathrm{d} \text { prednisolone, BV, } \\
\text { Ec-MS/BS, Ec-BFR, Ps-MS/BS, Ps-BFR at } 4 \\
\text { mg/kg/d prednisolone) at mid femur and } \\
\text { by histomorphometry }\end{array}$ & $\begin{array}{c}\text { Yes (maximum load }(\mathrm{N}) \text {, apparent ultimate } \\
\text { stress }(\mathrm{Mpa}) \text {, at } 4 \mathrm{mg} / \mathrm{kg} / \mathrm{d} \text { prednisolone } \\
\text { apparent toughness }\left(\mathrm{kj} / \mathrm{m}^{2}\right) \text { at } 4 \mathrm{mg} / \mathrm{kg} / \mathrm{d} \\
\text { prednisolone)at vertebral; (apparent } \\
\text { ultimate stress }(\mathrm{Mpa}) \text {, apparent toughness } \\
\left.\left(\mathrm{kj} / \mathrm{m}^{2}\right)\right) \text { at } 4 \mathrm{mg} / \mathrm{kg} / \mathrm{d} \text { prednisolone at } \\
\text { femur }\end{array}$ \\
\hline $\begin{array}{l}2011 \text { M Marenzana } \\
\text { [77] }\end{array}$ & Yes (BMD) at femur & $\begin{array}{c}\text { Yes (Tb.Th, Tb.N) at distal femur TB.Th, } \\
\text { longitudinal length) at L5 vertebra (Ct } \\
\text { volume, metaphysis Tb.Th) at femur } \\
\text { by } \mu \mathrm{CT}\end{array}$ & $\begin{array}{l}\text { Yes at femur (maximum load (N), ultimate } \\
\text { strength) }\end{array}$ \\
\hline $\begin{array}{l}2011 \text { H Henneicke } \\
\text { [78] }\end{array}$ & NA & $\begin{array}{c}\text { Yes (Tb.Th, Ct.Th, CT.Ar, MAR, BFR, } \\
\text { pericortical Area, osteoclast/pericortical } \\
\text { surface, pericortical area) at tibia by } \mu \mathrm{CT} \\
\text { and by histomorphometry }\end{array}$ & $\begin{array}{l}\text { No statistical difference reported in } \\
\text { mechanical load (N) and elastic modulus } \\
(\mathrm{Mpa})\end{array}$ \\
\hline 2014 Z Yongtao [26] & $\begin{array}{l}\text { Yes (BMD at } 12 \text { weeks } \\
\text { treatment) at L3-L4 vertebrae }\end{array}$ & $\begin{array}{c}\text { Yes (BV/TV, Tb.Th, MS/BS, MAR, BFR/BS, } \\
\text { N.Oc/BS, Oc/BS, ES/BS) at L3 vertebra } \\
\text { by histomorphometry }\end{array}$ & $\begin{array}{l}\text { Yes at } \mathrm{L} 4 \text { vertebra (maximum load }(\mathrm{N}) \text {, } \\
\text { stiffness }(\mathrm{N} / \mathrm{mm}) \text {, fracture stress }\left(\mathrm{N} / \mathrm{mm}^{2}\right) \text { ) }\end{array}$ \\
\hline 2011 M Ding [27] & NA & $\begin{array}{c}\text { Yes (CT.Po, bone surface: volume ratio, } \\
\text { bone surface density, cross sectional area } \\
\text { followed by } 3 \text { months without treatment) at } \\
\text { midshaft femur by } \mu \mathrm{CT}\end{array}$ & $\begin{array}{l}\text { No statistical difference measured in } \\
\text { ultimate stress }(\mathrm{MPa}) \text {, ultimate strain }(\%) \\
\text { Young's modulus }(\mathrm{GPa}) \text {, failure energy } \\
\qquad\left(\mathrm{kJ} / \mathrm{cm}^{3}\right) \text { at femur }\end{array}$ \\
\hline
\end{tabular}

\section{Discussion}

\subsection{Characteristics of Species and Strains}

The present systematic review provides an overview of animal models of GIOP. We found large heterogeneity in both methods applied to its establishment and in the animals used. Hence, the types of GC treatment, periods of administration, dose frequency, and administration route differ from study to study (Tables 2-7). We limited our investigation to murinae, sheep, and rabbit. However, there is still heterogeneity in the models related to different species, strain, age, and genders used in the experimental protocol (Tables 2-7).

Research into postmenopausal osteoporosis contributed to the use of rats as a reference animal model based on FDA guidelines [79], which have been widely published [16,17]. In the present work, $66 \%$ of the papers reviewed used rats, of which $77 \%$ involved Sprague Dawley (17\% Wistar and 6\% others). However, rats show some metabolic differences compared to human bone. The rat bone architecture does not have a Haversian system and some long bones retain their longitudinal growth capacity for most of their lives. Thus, beyond 30 months, the growth epiphyses of rats remain open [17]. In cancellous bones such as the lumbar vertebrae, the main activity before three months is bone shaping and not remodeling. These characteristics should be considered in dynamic histomorphometry analyses.

Although rats for many years have constituted a model of choice for bone studies, genetic engineering and biological tools remain limited for the study of this species. Conversely, mice who share more than $95 \%$ of their genome with the human species can be easily modified to create disease-specific knockouts. Mice in GIOP show a similar pattern of bone loss to humans, with an early phase of accelerated bone resorption followed by a slower phase of inhibited bone formation [80], but this is strain dependent [65]. In fact, it has been shown that mice with a $\mathrm{C} 57 \mathrm{Bl} / 6$ genetic background have a lower susceptibility to GIOP than CD1 strains [65].

Because of their size, sheep are the preferred model for research into the treatment of osteoporotic fracture and for studies on biomaterials for medical devices. The most 
commonly used osteoporotic sheep model is the ovariectomized sheep, but this model alone appears to have moderate impacts on bone mass as the majority of studies combine the use of OVX with GC treatment [81]. However, models of osteoporosis induced by GCs alone appear to approximate bone conditions comparable to those found in steroid-treated humans with changes in microarchitecture and mechanical properties [13,82]. Only one study [27] investigated GIOP in a purely GCs induced osteoporosis protocol (without ovariectomy) that showed an increase in the cortical porosity. However, this study combined the effects of GCs with a diet low in calcium and phosphorus, which is a source of bias in the study of bone loss due to steroid therapy alone.

Adult rabbits have a Haversian system and reach skeletal maturity. In addition, they have a high turnover rate with remodeling predominating over the shaping process, all conditions that can be considered as promising to constitute a model for osteoporosis research. In addition, rabbits are sexually mature at 6-8 months of age and exhibit closure of the growth epiphyses [83]. However, achieving significant bone loss in rabbits necessitates concomitant ovariectomy [84].

Rats and mice are the most commonly used species in GIOP animal studies. These animals are easy to breed, house, and manipulate and have a relatively low operating cost, unlike large animals such as sheep.

\subsection{Molecules, Dose, Duration, and Route of Administration}

Several GCs have been used in animal models of GIOP (Figure 2). Although, DEX is the most widely used corticosteroid in rats and prednisolone is the most widely used corticosteroid in mice, it is difficult to consider them as the reference GCs to be used in animal models of GIOP as other molecules such as prednisone and MP appear to be effective in inducing GIOP (Table 10). However, these corticosteroids do not have the same glucocorticoid potency as their natural counterpart, hydrocortisone. Their mineralocorticoid activity is negligible. DEX has 25 times the activity of hydrocortisone, MP five times, and prednisolone four times [85]. The potency of GCs has been studied in a single protocol that compared the effects of DEX and MP (at a dose of $0.6 \mathrm{mg} / \mathrm{kg}$ twice weekly and $1 \mathrm{mg} / \mathrm{kg}$ once daily, respectively) but for the same duration (12 weeks), the same route of administration, and the same strain [41]. In this study, the loss of BMD measured on the L1-L3 vertebrae was greater in the DEX group compared to the MP group [41]. Interestingly, in another study aiming at comparing DEX (200 $\mu \mathrm{g} / \mathrm{kg} 5$ day a week IP) vs. MP ( $5 \mathrm{mg} / \mathrm{kg} 5$ day a week SC) in Sprague Dawley female rats with the same duration, the comparison could not be performed due to a high rate of death in the DEX group. However, the dose used in this protocol was lower than the usual doses reported in other papers using Sprague Dawley females (Table 2).

It should be noted that the relative efficacy of GCs may vary depending on the route of administration and dosage, and that the effects may differ depending on the site of measurement. One study investigated the dose effect with prednisone on bone metabolism using different doses (1.5, 3, or $6 \mathrm{mg} / \mathrm{kg} /$ day for 90 days P.O.) in male Sprague Dawley rats [31]. The results showed that for a dose lower than $6 \mathrm{mg} / \mathrm{kg}$, the measurement of Tb.Th at the proximal tibia was not significantly different from the control group. However, for the same dose $(6 \mathrm{mg} / \mathrm{kg})$, the result of $\mathrm{Tb}$. Th was not significantly different from the control at the femur, illustrating the fact that for the same dose, the sensitivity to GCs could be different according to the bone site of interest.

In clinical routine, the main routes of administration are P.O, IM, and IV [86]. To mimic GIOP, it would therefore be logical to follow these routes of administration. However, our studies have highlighted other routes of administration such as the IP and PSC. 
Table 10. Studies responding to our definition of a good GIOP animal model. SD = Sprague Dawley.

\begin{tabular}{|c|c|c|c|c|}
\hline References & GCs Used & BMD Loss & $\begin{array}{l}\text { Alteration of the } \\
\text { Microarchitecture }\end{array}$ & $\begin{array}{c}\text { Decrease in } \\
\text { Biomechanical Properties }\end{array}$ \\
\hline 2019 Y Xu [33] Rat female SD & $\begin{array}{c}\text { DEX (2.5 mg/kg twice per week } \\
\text { for } 2 \text { months, IM) }\end{array}$ & Femur & Femur & Femur \\
\hline 2017 H Ren [36] Rat female SD & $\begin{array}{c}\text { DEX (0.6 mg } / \mathrm{kg} \text { every } 3 \text { days for } 3 \\
\text { months, SC) }\end{array}$ & L1-L5 vertebrae & L2 vertebra & L2 vertebra \\
\hline $\begin{array}{c}2017 \text { M Zhou [37] Rat female } \\
\text { SD }\end{array}$ & $\begin{array}{c}\text { Prednisone }(6 \mathrm{mg} / \mathrm{kg} \text { daily for } \\
21 \text { weeks, SC) }\end{array}$ & $\begin{array}{l}\text { L4 vertebra and } \\
\text { femur }\end{array}$ & Femur & Femur \\
\hline $\begin{array}{l}2017 \text { G Chen [38] Rat female } \\
\text { SD }\end{array}$ & $\begin{array}{c}\text { Prednisone ( } 5 \mathrm{mg} / \mathrm{kg} \text { daily for } 90 \\
\text { days, P.O) }\end{array}$ & Femur & Tibia & Femur \\
\hline $\begin{array}{l}2016 \text { G Shen [40] Rat female } \\
\text { SD }\end{array}$ & $\begin{array}{l}\text { DEX (0.6 mg/kg twice per week } \\
\text { for } 3 \text { months, SC) }\end{array}$ & L1-L3 vertebrae & L4 vertebra & L4 vertebra \\
\hline 2015 H Ren [41] Rat female SD & $\begin{array}{c}\operatorname{DEX}(0.6 \mathrm{mg} / \mathrm{kg} \text { daily for } \\
12 \text { weeks, SC) MP }(1 \mathrm{mg} / \mathrm{kg} \text { daily } \\
\text { for } 12 \text { weeks, SC) }\end{array}$ & L1-L3 vertebrae & L4 vertebra & L4 vertebra \\
\hline $\begin{array}{c}2017 \text { G Pizzino [43] Rat female } \\
\text { SD }\end{array}$ & MP (30 mg/kg for 60 days, SC) & Femur & Femur and vertebra & Femur \\
\hline $\begin{array}{c}2016 \text { D Liang [45] Rat female } \\
\text { SD }\end{array}$ & $\begin{array}{c}\text { DEX }(0.6 \mathrm{mg} / \mathrm{kg} \text { twice per week or } \\
\text { daily for } 3 \text { months, IM) }\end{array}$ & L1-L4 vertebrae & L2 vertebra & L2 vertebra \\
\hline 2015 Y Liu [46] Rat female SD & $\begin{array}{c}\text { DEX (2 mg/kg twice per week for } \\
12 \text { weeks, IM) }\end{array}$ & $\begin{array}{l}\text { Femur and L5 } \\
\text { vertebra }\end{array}$ & Tibia & Femur \\
\hline 2013 Z Ren [50] Rat male SD & $\operatorname{DEX}(0.1 \mathrm{mg} / \mathrm{kg}$ daily for 5 weeks, & Femur & Femur & Femur \\
\hline $\begin{array}{l}2013 \text { F-S Wang [51] Rat male } \\
\text { SD }\end{array}$ & $\begin{array}{c}\mathrm{MP} \text { (5 mg/kg daily for } 1-2 \text { or } \\
4 \text { weeks, SC) }\end{array}$ & Femur & Femur & Yes at femur (Load $(\mathrm{N}))$ \\
\hline $\begin{array}{l}2011 \text { F-S Wang [52] Rat male } \\
\text { SD }\end{array}$ & $\begin{array}{c}\mathrm{DEX}(0.1 \mathrm{mg} / \mathrm{kg} \text { daily for } 1-2 \text { or } \\
5 \text { weeks, SC) }\end{array}$ & $\begin{array}{l}\text { Bone site no } \\
\text { reported }\end{array}$ & Tibia & Bone site no reported \\
\hline 2012 L Cui [53] Rat male SD & $\begin{array}{c}\text { Prednisone }(3.5 \mathrm{mg} / \mathrm{kg} \text { daily for } \\
12 \text { weeks, P.O) }\end{array}$ & Femur & Tibia & Femur \\
\hline 2017 Y Yang [54] Rat male SD & $\begin{array}{c}\text { DEX (1 mg/ } \mathrm{kg} \text { twice per week for } \\
8 \text { weeks, IM) }\end{array}$ & Femur & Femur & Femur \\
\hline 2014 S Lin [31] Rat male SD & $\begin{array}{c}\text { Prednisone }(1.5 \text { or } 3 \text { or } 6 \mathrm{mg} / \mathrm{kg} \\
\text { daily for } 90 \text { days, P.O) }\end{array}$ & Femur & $\begin{array}{l}\text { Tibia at } 6 \mathrm{mg} / \mathrm{kg} \text { and } \\
\text { femur at } 3 \mathrm{mg} / \mathrm{kg}\end{array}$ & $\begin{array}{l}\text { Femur and L5 vertebra at } \\
\qquad 6 \mathrm{mg} / \mathrm{kg}\end{array}$ \\
\hline 2012 J-Y Ko [55] Rat male SD & 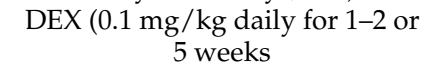 & $\begin{array}{l}\text { Bone site no } \\
\text { reported }\end{array}$ & Bone site no reported & Tibia \\
\hline $\begin{array}{l}2020 \text { D Sato [57] Rat female } \\
\text { LEW CrlCrlj }\end{array}$ & $\begin{array}{l}\text { Prednisolone ( } 0.42 \mathrm{mg} \text { daily for } \\
6 \text { weeks, PSC) }\end{array}$ & Femur & Tibia & Tibia \\
\hline $\begin{array}{c}2016 \text { A Y Sato [64] Mice female } \\
\text { C57BL/6 }\end{array}$ & $\begin{array}{c}\text { Prednisolone }(1.4 \text { or } 2.1 \mathrm{mg} / \mathrm{kg} \\
\text { daily for } 90 \text { days, PSC) }\end{array}$ & $\begin{array}{l}\text { Whole body and } \\
\text { L1-L6 vertebrae }\end{array}$ & L1-L3 vertebrae & L6 vertebra \\
\hline $\begin{array}{c}2019 \text { Q Geng [66] Mice male } \\
\text { C57BL/6 }\end{array}$ & $\begin{array}{c}\text { DEX (1 mg/kg for } 5 \text { days per week } \\
\text { for } 4 \text { weeks, } \mathrm{SC})\end{array}$ & $\begin{array}{l}\text { Total body, } \\
\text { vertebra and } \\
\text { femur }\end{array}$ & Femur & Femur \\
\hline $\begin{array}{l}2018 \text { I Alam [74] Mice male } \\
\text { and female Col2.3hWNT16TG }\end{array}$ & $\begin{array}{l}\text { Prednisolone }(2.1 \mathrm{mg} / \mathrm{kg} \text { daily for } \\
28 \text { days, PSC) }\end{array}$ & Femur & Femur & Femur \\
\hline $\begin{array}{c}2011 \text { M Marenzana [77] Mice } \\
\text { BALB/c }\end{array}$ & $\begin{array}{c}\operatorname{DEX}(3 \mathrm{mg} / \mathrm{kg} \text { daily for } 6 \text { or } \\
9 \text { weeks, P.O) }\end{array}$ & Femur & $\begin{array}{l}\text { Femur and } \\
\text { L5 vertebra }\end{array}$ & Femur \\
\hline $\begin{array}{l}2014 \text { Z Yongtao [26] Rabbit } \\
\text { male New Zealand white }\end{array}$ & $\begin{array}{c}\text { DEX (3 mg/kg twice per week for } \\
12 \text { weeks, IM) }\end{array}$ & L3-L4 vertebrae & L3 vertebra & L4 vertebra \\
\hline
\end{tabular}

Daily injections are stressful for the animals, which may interfere with the study and bias the results. Repeated injections have been shown to increase serum corticosterone levels one hour after injection in mice [87]. We observed in mice that those of PSC were predominantly employed ( $66 \%$ of studies in mice). However, the use of PSC involves surgery, which requires increased attention to animals and more hover increases the risk of postoperative infection. The majority of the studies in this work reported that GCs were given by injection and not P.O, which is opposite to human clinical practice [86]. However, the way in which the P.O was given was not always explained. Administration can be by capsule in the feed, dissolved in the drinking water or by gavage to ensure absorption of the GCs, but the latter method induces additional stress for the animal [88].

There was also high heterogeneity in the duration of GC treatment in the studies analyzed in the present review. In Sprague Dawley male DXA, analyses showed that GC treated rats (MP $5 \mathrm{mg} / \mathrm{kg}$ daily $\mathrm{SC}$ ) had lower BMD and BMC than the vehicle-treated group as soon as after two weeks of treatment. However, it needs four weeks of GC treatment to observe a reduced metaphyseal trabecular microstructure [51]. In two other studies, 
the same strain and sex (male Sprague Dawley) rats were treated with $0.1 \mathrm{mg} / \mathrm{kg} / \mathrm{day}$ SC DEX for one, two, or five weeks. The $\mu \mathrm{CT}$ measurements showed that the rats had more metaphyseal trabecular bone loss than the vehicle-treated group as soon as five weeks of treatment [52,55]. DXA analyses showed a reduction in BMD at both two [55] and five weeks [52,55]. These findings illustrated the different sensitivity of the two devices (DXA, $\mu \mathrm{CT}$ ) to show the GC effects and could give some information about the minimal length of treatment to induce GIOP. However, the aforementioned studies seem to show that DXA measurement should evidence a bone decrease earlier than with $\mu \mathrm{CT}$ measurement in Sprague Dawley rats, which cannot be extrapolated to other animals. Hence, in female six week old C57BL/ 6 mice, TB.Th measured by $\mu$ CT was significantly decreased at both distal femur and proximal tibia after 14 days of prednisolone $2.1 \mathrm{mg} / \mathrm{kg} / \mathrm{d}$, but a significant decrease in BMD (measured by DXA) at the femur and spine was only observed after 28 days of treatment [63].

\subsection{Impact of the Weight, Age, and Sex on the Establishment of GIOP Model}

GCs in animal models affect both body weight and size. Thus, theoretically when measuring GC effects on bone loss, bone size adjustments should be performed to limit bias in interpreting the effect on BMD [89]. It appears that none of the studies found in this review reported an adjustment for bone size in the section on statistical analysis.

Conversely, findings from a study using prednisone in young rats (three months) reported data of Lin et al. in 2014 [31], indicating that prednisone reduced bone growth, which raises the question of whether it is appropriate to use rats that are barely skeletally mature. Rats reach sexual maturity at about six weeks of age, but their bodies including their skeletons are constantly growing. Rats are considered adult when they are socially mature, which is six months later [90]. It is accepted that one month of rat life corresponds to 2.5 years of human life [91].

Gender and strain-specific efficacy of GCs have been previously demonstrated [92,93]. The effects of prednisone (6 mg/kg/day P.O) in male and female Sprague Dawley rats were investigated on bone parameters for 21 weeks [37]. Findings demonstrated that the BV/TV parameter decreased by $52 \%$ and $27.0 \%$ at the femur and L4 vertebra, respectively, in females, whereas in males, the loss was $28.6 \%$ and $14.0 \%$ at the femur and L4, respectively. Unfortunately, in a study conducted in male and female Wistar rats to demonstrate the efficacy of a Chinese herbal medicine in GIOP, no comparison was undertaken to compare males and females concerning the results on bone parameters [30].

\subsection{Methods of Measurement of the Establishment of the GIOP Bone Phenotype}

Laboratory techniques are used in GIOP animal models and inside these techniques, their nuances are one of the key issues related to the appropriate methodology to evaluate animal models of GIOP and extrapolate findings from animals to humans.

In the present systematic review, we deliberately decided to report original articles using either dual-energy X-ray absorptiometry (DXA) or micro-computed tomography $(\mu \mathrm{CT})$ to assess areal BMD or volumetric BMD to demonstrate GIOP bone loss. We also chose to consider articles that evaluated the effects of GCs on bone microarchitecture either by $\mu \mathrm{CT}$ or by quantitative histological techniques. In addition, to appreciate the most appropriate GIOP models, we sought to select studies that evaluated the bone biomechanical properties. Indeed, in order to actually mimic GIOP in humans, we considered that a relevant animal model of GIOP would produce both bone loss and alterations in the microarchitecture, but also decrease the bone biomechanical properties.

DXA is considered the gold standard for the diagnosis of human osteoporosis. This technique is also widely used in rodents and provide assessments of bone mass and gross morphology in and ex vivo. Although measurements were performed on small bones or on the whole body, the DXA measurement was accurate and precise. However, this technique is limited by its low spatial resolution; moreover, the accuracy of this technique cannot permit compartment specific bone parameter analyses. However, DXA and peripheral 
quantitative $\mathrm{CT}$ produced comparable results in terms of precision, accuracy, and sensitivity to change when examining the rat femur [94].

Conversely, $\mu \mathrm{CT}$ is currently considered as the gold standard for the assessment of vBMD and microarchitecture, both at the trabecular and cortical bone [22]. Furthermore, new devices provide the opportunity of in vivo scanning that permit longitudinal analyses such as the monitoring of bone metastasis [95] or bio-integration of bone implants [96]. In the present systematic review, $64 \%$ of the studies were conducted with ex vivo $\mu \mathrm{CT}$ and $1 \%$ with the functionality of in vivo scanning.

Histological analyses are very important because they provide both static and dynamic histomorphometric parameters and information at the tissue and cellular levels. Using this method, both cancellous bone (at lumbar vertebral bodies, tibia, or femur metaphysis) and the cortical bone can be investigated. The cortical bone is mainly assessed at the diaphysis of the long bone.

However, even in the best GIOP animal models, spontaneous fracture such as in humans does not occur. Consequently, biomechanical testing has been considered in GIOP animal models as a reliable surrogate marker of bone fragility [97,98].

Bone strength is usually evaluated through the three-point bending test of long bone (femur or tibia) and axial compression tests on vertebral bodies. However, this review provides evidences that a number of biomechanical properties were reported, hence, more than forty different parameters were provided, as indicated in Table 9. The heterogeneities in the methods justify the demand of consensus regarding both extrinsic and intrinsic biomechanical parameter methods are reported in the literature. Mechanical tests in three-point bending and compression determine the actual strength of the bone material. Depending on the load applied, the stresses can be compressive, tensile, or shear forces. The parameters measured reveal the extrinsic (structural) forces of the bone: ultimate load $(\mathrm{N})$, stiffness $(\mathrm{N} / \mathrm{mm})$, yield load $(\mathrm{N})$, elastic energy (N.mm), and plastic energy (N.mm). The intrinsic force can be described by the following parameters: Young's modulus (modulus of elasticity $\mathrm{MPa})$, toughness $\left(\mathrm{MJ} / \mathrm{m}^{3}\right)$, and ultimate stress $\left(\mathrm{N} / \mathrm{mm}^{2}\right)$ as defined by Turner and Draca $[24,25]$. Sometimes, solely the units of bone biomechanical properties have been presented without a full description of the parameter evaluated.

It is well known that GIOP patients fracture at higher BMD values than in postmenopausal osteoporosis. In clinical practice, fracture is most often associated with altered density and micro-architecture; however, in GIOP, the epidemiological data seem to show that this relationship is not always true [99]. Interestingly, only one study in our review reported a decrease in bone strength (maximum load $(\mathrm{N})$ and energy $(\mathrm{mJ})$ ) measured at the femur while there was no significant decrease in BMD measured at the femur [62].

Finally, several studies are in accordance with the present good model definition of GIOP (i.e., a loss of bone density) with an alteration in the micro-architecture that leads to a decrease in the biomechanical strength of the bone (Table 10).

\subsection{Methodological Quality}

We also analyzed the methodological quality of all eligible reports using the definition by Schulz et al. [21] and adapted by Perel et al. [100]. In those animal research studies $[21,100]$, the emphasis was placed on random allocation to group, adequate allocation concealment, and blinded assessment of outcome.

Allocation of random animals to experimental groups was performed in two thirds of the eligible studies and only eight studies $[42,43,57,61,63,64,69,71]$ performed a blinded assessment of outcomes. Furthermore, this blinded assessment was only conducted for histomorphometric measurements. Methods for adequate allocation concealment were not clearly reported in the studies analyzed in our work. This lack of adequate allocation concealment has already been evidenced in both antenatal corticosteroids and corticosteroids for traumatic head injury studies where this was reported in $0 \%$ and $18 \%$ of the studies, respectively [100]. 
In addition, age, gender, weight, techniques of P.O administration of GCs, and number of animals were not systematically reported in some studies.

Finally, we did not find any study reporting the a priori sample size calculation of animals in the statistical methods section.

However, we acknowledge that authors might have undergone good quality procedure in conducting their study without correctly reporting it in their article.

\subsection{Strength and Limitation}

The strength of the current review is warranted by our systematic approach to search for original articles in one of the major online databases (i.e., PubMed).

All papers identified by the search strategy were retrievable and analyzed. However, we acknowledge that there are some limitations to consider. First, we limited our search strategy to one database, thus articles not indexed in PubMed were not included and reviewed even though they could potentially be relevant for our purpose. Second, we limited our search strategy to the last decade and focused our work on a limited number of species, omitting models using dogs or emerging zebra fish models [101,102].

Nevertheless, 53 full text articles were analyzed.

It is widely accepted that the underlying inflammatory disease that require GCs has a role in the pathophysiology of GIOP in human [103]. However, in our protocol, we decided to exclude studies that designed their methodological protocols including a previous induction of a glucocorticoid requiring disease.

Attempts should be made to standardize pre-clinical GIOP animal models. Such guidelines would enhance cross-laboratory comparisons and avoid the selection of the animal model, which could be driven more by convenience or past experience rather than based on evidence.

We have to underline that in the ideal, GIOP animal models would exhibit disease characteristics that are comparable to the human conditions. However, first, none of the eligible studies designed their methodological protocols including a previous induction of a glucocorticoid requiring disease. Indeed, it is widely accepted that the underlying inflammatory disease that requires GCs has a role in the pathophysiology of GIOP in humans [103]. This is a limitation of our study since we did not want to analyze the combined effects of corticosteroids on bone with pathology or treatments that could induce a bone phenotype.

Furthermore, we only found one study [45] that proposed, as in the human GIOP clinical trials [104], a designation of both treatment and prevention.

\section{Conclusions}

Direct comparison among studies is challenging due to the heterogeneity of the various experimental designs reported in relation to the dose, the route of administration, the duration, the type of GCs, and possibly the different bioavailabilities of the different GCs used as a function of the animal species. Nevertheless, we showed that the use of DEX in Sprague Dawley rats and prednisolone in mice are the most popular GIOP models, and that the dose and duration of these last GCs should be decided according to the age of the animal and the bone site chosen to be assessed. The results of this systematic review suggested that it lacks studies on the length of the persistence of the deleterious effects of GCs on bone tissue. Such studies might improve the management in terms of duration of therapies against GIOP. Although the present systematic review provides relevant information on the animal literature in GIOP, it also points to the poor quality of some protocols and suggest the need for guidelines in GIOP animal models to improve standardization and research outcomes aiming at the establishment of a better predictability of animal research in further human clinical trials.

Author Contributions: Conceptualization, A.X. and E.L.; Methodology, A.X. and E.L.; Validation, A.X., H.T. and E.L.; Formal analysis, A.X. and E.L.; Investigation, A.X. and E.L.; Writing-original draft preparation, A.X. and E.L.; Data curation, A.X. and E.L.; Writing-review and editing, A.X., H.T. 
and E.L.; Supervision, A.X. and E.L.; Project administration, A.X. and E.L. All authors have read and agreed to the published version of the manuscript.

Funding: This research received no external funding.

Institutional Review Board Statement: Not applicable.

Informed Consent Statement: Not applicable.

Data Availability Statement: The data presented in this study are available on request from the corresponding author.

Acknowledgments: The authors would like to thank Julie BRUGNEAUX at Orleans Hospital for her help in the search strategy for articles in the PubMed database.

Conflicts of Interest: The authors declare no conflict of interest.

\section{References}

1. Briot, K.; Roux, C. Glucocorticoid-Induced Osteoporosis. RMD Open 2015, 1, e000014. [CrossRef]

2. Compston, J. Glucocorticoid-Induced Osteoporosis: An Update. Endocrine 2018, 61, 7-16. [CrossRef]

3. Amiche, M.A.; Albaum, J.M.; Tadrous, M.; Pechlivanoglou, P.; Lévesque, L.E.; Adachi, J.D.; Cadarette, S.M. Fracture Risk in Oral Glucocorticoid Users: A Bayesian Meta-Regression Leveraging Control Arms of Osteoporosis Clinical Trials. Osteoporos. Int. 2016, 27, 1709-1718. [CrossRef]

4. Lespessailles, E.; Chapurlat, R. High Fracture Risk Patients with Glucocorticoid-Induced Osteoporosis Should Get an Anabolic Treatment First. Osteoporos. Int. 2020, 31, 1829-1834. [CrossRef] [PubMed]

5. Canalis, E.; Mazziotti, G.; Giustina, A.; Bilezikian, J.P. Glucocorticoid-Induced Osteoporosis: Pathophysiology and Therapy. Osteoporos. Int. 2007, 18, 1319-1328. [CrossRef] [PubMed]

6. Lane, N.E.; Yao, W. New Insights into the Biology of Glucocorticoid-Induced Osteoporosis Introduction-The Clinical Importance of Glucocorticoid-Induced Bone Loss. IBMS BoneKEy 2011, 8, 229-236. [CrossRef]

7. De Vries, F.; Bracke, M.; Leufkens, H.G.M.; Lammers, J.W.J.; Cooper, C.; Van Staa, T.P. Fracture Risk with Intermittent High-Dose Oral Glucocorticoid Therapy. Arthritis Rheum. 2007, 56, 208-214. [CrossRef]

8. Van Staa, T.P.; Leufkens, H.G.M.; Abenhaim, L.; Zhang, B.; Cooper, C. Use of Oral Corticosteroids and Risk of Fractures. J. Bone Miner. Res. 2000, 15, 993-1000. [CrossRef]

9. Adami, G.; Saag, K.G. Glucocorticoid-Induced Osteoporosis: 2019 Concise Clinical Review. Osteoporos. Int. 2019, 30, 1145-1156. [CrossRef]

10. Adamopoulos, I.E. Inflammation in Bone Physiology and Pathology. Curr. Opin. Rheumatol. 2018, 30, 59-64. [CrossRef]

11. Cafiero, C.; Gigante, M.; Brunetti, G.; Simone, S.; Chaoul, N.; Oranger, A.; Ranieri, E.; Colucci, S.; Pertosa, G.B.; Grano, M.; et al. Inflammation Induces Osteoclast Differentiation from Peripheral Mononuclear Cells in Chronic Kidney Disease Patients: Crosstalk between the Immune and Bone Systems. Nephrol. Dial. Transplant. 2018, 33, 65-75. [CrossRef]

12. Buckley, L.; Guyatt, G.; Fink, H.A.; Cannon, M.; Grossman, J.; Hansen, K.E.; Humphrey, M.B.; Lane, N.E.; Magrey, M.; Miller M.; et al. 2017 American College of Rheumatology Guideline for the Prevention and Treatment of Glucocorticoid-Induced Osteoporosis. Arthritis Rheumatol. 2017, 69, 1521-1537. [CrossRef]

13. Oheim, R.; Amling, M.; Ignatius, A.; Pogoda, P. Large Animal Model for Osteoporosis in Humans: The Ewe. Eur. Cells Mater. 2012, 24, 372-385. [CrossRef]

14. Reinwald, S.; Burr, D. Review of Nonprimate, Large Animal Models for Osteoporosis Research. J. Bone Miner. Res. 2008, 23, 1353-1368. [CrossRef] [PubMed]

15. R Brommage Perspectives on Using Nonhuman Primates to Understand the Etiology and Treatment of Postmenopausal Osteoporosis-PubMed. Available online: https:/ / pubmed.ncbi.nlm.nih.gov/15758482/ (accessed on 18 June 2021).

16. Komori, T. Animal Models for Osteoporosis. Eur. J. Pharmacol. 2015, 759, 287-294. [CrossRef] [PubMed]

17. Lelovas, P.P.; Xanthos, T.T.; Thorma, S.E.; Lyritis, G.P.; Dontas, I.A. The Laboratory Rat as an Animal Model for Osteoporosis Research. Comp. Med. 2008, 58, 424-430. [PubMed]

18. Page, M.J.; McKenzie, J.E.; Bossuyt, P.M.; Boutron, I.; Hoffmann, T.C.; Mulrow, C.D.; Shamseer, L.; Tetzlaff, J.M.; Akl, E.A.; Brennan, S.E.; et al. The PRISMA 2020 Statement: An Updated Guideline for Reporting Systematic Reviews. BMJ 2021, $372,377$.

19. Kalu, D.N. The Ovariectomized Rat Model of Postmenopausal Bone Loss. Bone Miner. 1991, 15, 175-191. [CrossRef]

20. Turner, R.T.; Maran, A.; Lotinun, S.; Hefferan, T.; Evans, G.L.; Zhang, M.; Sibonga, J.D. Animal Models for Osteoporosis. Rev. Endocr. Metab. Disord. 2001, 2, 117-127. [CrossRef]

21. Schulz, K.F.; Chalmers, I.; Hayes, R.J.; Altman, D.G. Empirical Evidence of Bias: Dimensions of Methodological Quality Associated With Estimates of Treatment Effects in Controlled Trials. JAMA J. Am. Med. Assoc. 1995, 273, 408-412. [CrossRef]

22. Bouxsein, M.L.; Boyd, S.K.; Christiansen, B.A.; Guldberg, R.E.; Jepsen, K.J.; Müller, R. Guidelines for Assessment of Bone Microstructure in Rodents Using Micro-Computed Tomography. J. Bone Miner. Res. 2010, 25, 1468-1486. [CrossRef] [PubMed] 
23. Parfitt, A.M.; Drezner, M.K.; Glorieux, F.H.; Kanis, J.A.; Malluche, H.; Meunier, P.J.; Ott, S.M.; Recker, R.R. Bone Histomorphometry: Standardization of Nomenclature, Symbols, and Units. Report of the ASBMR Histomorphometry Nomenclature Committee. J. Bone Miner. Res. Off. J. Am. Soc. Bone Miner. Res. 1987, 2, 595-610. [CrossRef] [PubMed]

24. Turner, C.H.; Burr, D.B. Basic Biomechanical Measurements of Bone: A Tutorial. Bone 1993, 14, 595-608. [CrossRef]

25. Draca, N.; Tikvica, A.; Eljuga, D.; Semenski, D.; Brncić, M.; Vukicević, S. Biomechanical Properties of Bones from Rats Treated with Sevelamer-PubMed. Available online: https://pubmed.ncbi.nlm.nih.gov/21755732/ (accessed on 18 August 2021).

26. Yongtao, Z.; Kunzheng, W.; Jingjing, Z.; Hu, S.; Jianqiang, K.; Ruiyu, L.; Chunsheng, W. Glucocorticoids Activate the Local Renin-Angiotensin System in Bone: Possible Mechanism for Glucocorticoid-Induced Osteoporosis. Endocrine 2014, 47, 598-608. [CrossRef]

27. Ding, M.; Danielsen, C.C.; Overgaard, S. The Effects of Glucocorticoid on Microarchitecture, Collagen, Mineral and Mechanical Properties of Sheep Femur Cortical Bone. J. Tissue Eng. Regen. Med. 2012, 6, 443-450. [CrossRef]

28. Mo, Y.; Lai, W.; Zhong, Y.; Hu, Z.; You, M.; Du, M.; Wang, P.; Wu, X.; Chen, C.; He, H.; et al. TXNIP Contributes to Bone Loss via Promoting the Mitochondrial Oxidative Phosphorylation during Glucocorticoid-Induced Osteoporosis. Life Sci. 2021, $266,118938$. [CrossRef]

29. Chen, Z.; Xue, J.; Shen, T.; Ba, G.; Yu, D.; Fu, Q. Curcumin Alleviates Glucocorticoid-Induced Osteoporosis by Protecting Osteoblasts from Apoptosis in Vivo and in Vitro. Clin. Exp. Pharmacol. Physiol. 2016, 43, 268-276. [CrossRef]

30. Han, N.; Xu, J.; Xu, F.; Liu, Z.; Yin, J. The in Vivo Effects of a Fraction from Dioscorea Spongiosa on Glucocorticoid-Induced Osteoporosis. J. Ethnopharmacol. 2016, 185, 53-59. [CrossRef]

31. Lin, S.; Huang, J.; Zheng, L.; Liu, Y.; Liu, G.; Li, N.; Wang, K.; Zou, L.; Wu, T.; Qin, L.; et al. Glucocorticoid-Induced Osteoporosis in Growing Rats. Calcif. Tissue Int. 2014, 95, 362-373. [CrossRef]

32. Yao, W.; Dai, W.; Jiang, L.; Lay, E.Y.A.; Zhong, Z.; Ritchie, R.O.; Li, X.; Ke, H.; Lane, N.E. Sclerostin-Antibody Treatment of Glucocorticoid-Induced Osteoporosis Maintained Bone Mass and Strength. Osteoporos. Int. 2016, 27, 283-294. [CrossRef]

33. Xu, Y.; Guan, J.; Xu, J.; Chen, S.; Sun, G. Z-Guggulsterone Attenuates Glucocorticoid-Induced Osteoporosis through Activation of Nrf2/HO-1 Signaling. Life Sci. 2019, 224, 58-66. [CrossRef]

34. Zhao, J.; Li, Y.; Zhang, H.; Shi, D.; Li, Q.; Meng, Y.; Zuo, L. Preventative Effects of Metformin on Glucocorticoid-Induced Osteoporosis in Rats. J. Bone Miner. Metab. 2019, 37, 805-814. [CrossRef]

35. Yang, Y.J.; Zhu, Z.; Wang, D.T.; Zhang, X.L.; Liu, Y.Y.; Lai, W.X.; Mo, Y.L.; Li, J.; Liang, Y.L.; Hu, Z.Q.; et al. Tanshinol Alleviates Impaired Bone Formation by Inhibiting Adipogenesis via KLF15/PPAR $\gamma 32$ Signaling in GIO Rats. Acta Pharmacol. Sin. 2018, 39, 633-641. [CrossRef] [PubMed]

36. Ren, H.; Shen, G.; Tang, J.; Qiu, T.; Zhang, Z.; Zhao, W.; Yu, X.; Huang, J.; Liang, D.; Yao, Z.; et al. Promotion Effect of Extracts from Plastrum Testudinis on Alendronate against Glucocorticoid-Induced Osteoporosis in Rat Spine. Sci. Rep. 2017, 7, 10617. [CrossRef] [PubMed]

37. Zhou, M.; Li, J.; Wu, J.; Yang, Y.; Zeng, X.; Lv, X.; Cui, L.; Yao, W.; Liu, Y. Preventive Effects of Polygonum Multiflorum on Glucocorticoid-Induced Osteoporosis in Rats. Exp. Ther. Med. 2017, 14, 2445-2460. [CrossRef]

38. Chen, G.; Zhang, X.; Lin, H.; Huang, G.; Chen, Y.; Cui, L. Tanshinol Alleviates Osteoporosis and Myopathy in GlucocorticoidTreated Rats. Planta Med. 2017, 83, 1264-1273. [CrossRef] [PubMed]

39. Yang, Y.; Su, Y.; Wang, D.; Chen, Y.; Liu, Y.; Luo, S.; Wu, T.; Cui, L. Tanshinol Rescues the Impaired Bone Formation Elicited by Glucocorticoid Involved in KLF15 Pathway. Oxidative Med. Cell. Longev. 2016, 2016. [CrossRef] [PubMed]

40. Shen, G.; Ren, H.; Qiu, T.; Liang, D.; Wei, Q.; Tang, J.; Zhang, Z.; Yao, Z.; Zhao, W.; Jiang, X. Effect of Glucocorticoid Withdrawal on Glucocorticoid Inducing Bone Impairment. Biochem. Biophys. Res. Commun. 2016, 477, 1059-1064. [CrossRef] [PubMed]

41. Ren, H.; Liang, D.; Jiang, X.; Tang, J.; Cui, J.; Wei, Q.; Zhang, S.; Yao, Z.; Shen, G.; Lin, S. Variance of Spinal Osteoporosis Induced by Dexamethasone and Methylprednisolone and Its Associated Mechanism. Steroids 2015, 102, 65-75. [CrossRef]

42. Khan, M.P.; Mishra, J.S.; Sharan, K.; Yadav, M.; Singh, A.K.; Srivastava, A.; Kumar, S.; Bhaduaria, S.; Maurya, R.; Sanyal, S.; et al. A Novel Flavonoid C-Glucoside from Ulmus Wallichiana Preserves Bone Mineral Density, Microarchitecture and Biomechanical Properties in the Presence of Glucocorticoid by Promoting Osteoblast Survival: A Comparative Study with Human Parathyroid Hormone. Phytomedicine 2013, 20, 1256-1266. [CrossRef]

43. Pizzino, G.; Irrera, N.; Galfo, F.; Oteri, G.; Atteritano, M.; Pallio, G.; Mannino, F.; D’ Amore, A.; Pellegrino, E.; Aliquò, F.; et al. Adenosine Receptor Stimulation Improves Glucocorticoid-Induced Osteoporosis in a Rat Model. Front. Pharmacol. 2017, 8, 558. [CrossRef] [PubMed]

44. Jiang, Y.; Gou, H.; Wang, S.; Zhu, J.; Tian, S.; Yu, L. Effect of Pulsed Electromagnetic Field on Bone Formation and Lipid Metabolism of Glucocorticoid-Induced Osteoporosis Rats through Canonical Wnt Signaling Pathway. Evid.-Based Complementary Altern. Med. 2016, 2016. [CrossRef] [PubMed]

45. Liang, D.; Ren, H.; Qiu, T.; Shen, G.; Xie, B.; Wei, Q.; Yao, Z.; Tang, J.; Zhang, Z.; Jiang, X. Extracts from Plastrum Testudinis Reverse Glucocorticoid-Induced Spinal Osteoporosis of Rats via Targeting Osteoblastic and Osteoclastic Markers. Biomed. Pharmacother. 2016, 82, 151-160. [CrossRef]

46. Liu, Y.; Cui, Y.; Chen, Y.; Gao, X.; Su, Y.; Cui, L. Effects of Dexamethasone, Celecoxib, and Methotrexate on the Histology and Metabolism of Bone Tissue in Healthy Sprague Dawley Rats. Clin. Interv. Aging 2015, 10, 1245-1253. [CrossRef] [PubMed] 
47. Pal, S.; Mittapelly, N.; Husain, A.; Kushwaha, S.; Chattopadhyay, S.; Kumar, P.; Ramakrishna, E.; Kumar, S.; Maurya, R.; Sanyal, S.; et al. A Butanolic Fraction from the Standardized Stem Extract of Cassia Occidentalis L Delivered by a Self-Emulsifying Drug Delivery System Protects Rats from Glucocorticoid-Induced Osteopenia and Muscle Atrophy. Sci. Rep. 2020, 10, 195. [CrossRef]

48. Yang, L.; Yang, J.; Pan, T.; Zhong, X. Liraglutide Increases Bone Formation and Inhibits Bone Resorption in Rats with Glucocorticoid-Induced Osteoporosis. J. Endocrinol. Investig. 2019, 42, 1125-1131. [CrossRef] [PubMed]

49. Feng, M.; Zhang, R.; Gong, F.; Yang, P.; Fan, L.; Ni, J.; Bi, W.; Zhang, Y.; Wang, C.; Wang, K. Protective Effects of Necrostatin-1 on Glucocorticoid-Induced Osteoporosis in Rats. J. Steroid Biochem. Mol. Biol. 2014, 144, 455-462. [CrossRef]

50. Ren, Z.; Yang, L.; Xue, F.; Meng, Q.; Wang, K.; Wu, X.; Ji, C.; Jiang, T.; Liu, D.; Zhou, L.; et al. Yeast-Incorporated Gallium Attenuates Glucocorticoid-Induced Bone Loss in Rats by Inhibition of Bone Resorption. Biol. Trace Elem. Res. 2013, 152, 396-402. [CrossRef]

51. Wang, F.S.; Chung, P.C.; Lin, C.L.; Chen, M.W.; Ke, H.J.; Chang, Y.H.; Chen, Y.S.; Wu, S.L.; Ko, J.Y. MicroRNA-29a Protects against Glucocorticoid-Induced Bone Loss and Fragility in Rats by Orchestrating Bone Acquisition and Resorption. Arthritis Rheum. 2013, 65, 1530-1540. [CrossRef] [PubMed]

52. Wang, F.S.; Wu, R.W.; Ko, J.Y.; Tai, M.H.; Ke, H.C.; Yeh, D.W.; Wu, S.L.; Chen, M.W. Heat Shock Protein 60 Protects Skeletal Tissue against Glucocorticoid-Induced Bone Mass Loss by Regulating Osteoblast Survival. Bone 2011, 49, 1080-1089. [CrossRef]

53. Cui, L.; Li, T.; Liu, Y.; Zhou, L.; Li, P.; Xu, B.; Huang, L.; Chen, Y.; Liu, Y.; Tian, X.; et al. Salvianolic Acid b Prevents Bone Loss in Prednisone-Treated Rats through Stimulation of Osteogenesis and Bone Marrow Angiogenesis. PLoS ONE 2012, 7 , e34647. [CrossRef] [PubMed]

54. Yang, Y.; Nian, H.; Tang, X.; Wang, X.; Liu, R. Effects of the Combined Herba Epimedii and Fructus Ligustri Lucidi on Bone Turnover and TGF-B1/Smads Pathway in GIOP Rats. J. Ethnopharmacol. 2017, 201, 91-99. [CrossRef] [PubMed]

55. Ko, J.Y.; Wu, R.W.; Kuo, S.J.; Chen, M.W.; Yeh, D.W.; Ke, H.C.; Wu, S.L.; Wang, F.S. Cannabinoid Receptor 1 Mediates Glucocorticoid-Induced Bone Loss in Rats by Perturbing Bone Mineral Acquisition and Marrow Adipogenesis. Arthritis Rheum. 2012, 64, 1204-1214. [CrossRef]

56. Yang, Y.; Yu, T.; Tang, H.; Ren, Z.; Li, Q.; Jia, J.; Chen, H.; Fu, J.; Ding, S.; Hao, Q.; et al. Ganoderma Lucidum Immune Modulator Protein RLZ-8 Could Prevent and Reverse Bone Loss in Glucocorticoids-Induced Osteoporosis Rat Model. Front. Pharmacol. 2020, 11, 731. [CrossRef]

57. Sato, D.; Takahata, M.; Ota, M.; Fukuda, C.; Hasegawa, T.; Yamamoto, T.; Amizuka, N.; Tsuda, E.; Okada, A.; Hiruma, Y.; et al. Siglec-15-Targeting Therapy Protects against Glucocorticoid-Induced Osteoporosis of Growing Skeleton in Juvenile Rats. Bone 2020, 135, 115331. [CrossRef] [PubMed]

58. Hou, T.; Zhang, L.; Yang, X. Ferulic Acid, a Natural Polyphenol, Protects against Osteoporosis by Activating SIRT1 and NF-KB in Neonatal Rats with Glucocorticoid-Induced Osteoporosis. Biomed. Pharmacother. 2019, 120, 109205. [CrossRef] [PubMed]

59. Lucinda, L.M.F.; Aarestrup, B.J.V.; Reboredo, M.M.; Pains, T.D.A.; Chaves, R.Z.; Reis, J.E.P.; Louzada, M.J.Q.; Guerra, M.O. Evaluation of the Anti-Osteoporotic Effect of Ginkgo Biloba L. In Wistar Rats with Glucocorticoid-Induced-Osteoporosis by Bone Densitometry Using Dualenergy x-Ray Absorptiometry (DEXA) and Mechanical Testing. Anais da Academia Brasileira de Ciências 2017, 89, 2833-2841. [CrossRef]

60. Achiou, Z.; Toumi, H.; Touvier, J.; Boudenot, A.; Uzbekov, R.; Ominsky, M.S.; Pallu, S.; Lespessailles, E. Sclerostin Antibody and Interval Treadmill Training Effects in a Rodent Model of Glucocorticoid-Induced Osteopenia. Bone 2015, 81, 691-701. [CrossRef]

61. Pichler, K.; Loreto, C.; Leonardi, R.; Reuber, T.; Weinberg, A.M.; Musumeci, G. RANKL Is Downregulated in Bone Cells by Physical Activity (Treadmill and Vibration Stimulation Training) in Rat with Glucocorticoid-Induced Osteoporosis. Histol. Histopathol. 2013, 28, 1185-1196. [CrossRef]

62. Saito, M.; Marumo, K.; Ushiku, C.; Kato, S.; Sakai, S.; Hayakawa, N.; Mihara, M.; Shiraishi, A. Effects of Alfacalcidol on Mechanical Properties and Collagen Cross-Links of the Femoral Diaphysis in Glucocorticoid-Treated Rats. Calcif. Tissue Int. 2011, 88, 314-324. [CrossRef]

63. Sato, A.Y.; Richardson, D.; Cregor, M.; Davis, H.M.; Au, E.D.; McAndrews, K.; Zimmers, T.A.; Organ, J.M.; Peacock, M.; Plotkin, L.I.; et al. Glucocorticoids Induce Bone and Muscle Atrophy by Tissue-Specific Mechanisms Upstream of E3 Ubiquitin Ligases. Endocrinology 2017, 158, 664-677. [CrossRef]

64. Sato, A.Y.; Cregor, M.; Delgado-Calle, J.; Condon, K.W.; Allen, M.R.; Peacock, M.; Plotkin, L.I.; Bellido, T. Protection From Glucocorticoid-Induced Osteoporosis by Anti-Catabolic Signaling in the Absence of Sost/Sclerostin. J. Bone Miner. Res. 2016, 31, 1791-1802. [CrossRef] [PubMed]

65. Ersek, A.; Santo, A.I.E.; Vattakuzhi, Y.; George, S.; Clark, A.R.; Horwood, N.J. Strain Dependent Differences in GlucocorticoidInduced Bone Loss between C57BL/6J and CD-1 Mice. Sci. Rep. 2016, 6, 36513. [CrossRef]

66. Geng, Q.; Heng, K.; Li, J.; Wang, S.; Sun, H.; Sha, L.; Guo, Y.; Nie, X.; Wang, Q.; Dai, L.; et al. A Soluble Bone Morphogenetic Protein Type 1A Receptor Fusion Protein Treatment Prevents Glucocorticoid-Induced Bone Loss in Mice-PubMed. Available online: https:/ / pubmed.ncbi.nlm.nih.gov/31396331/ (accessed on 22 June 2021).

67. Mao, L.; Wang, F.; Li, Y.; Dai, Y.; Liu, Y.; Wang, J.; Xue, C. Oil from Antarctic Krill (Euphausia Superba) Facilitates Bone Formation in Dexamethasone-Treated Mice. Food Sci. Biotechnol. 2019, 28, 539-545. [CrossRef] [PubMed]

68. Fenton, C.G.; Doig, C.L.; Fareed, S.; Naylor, A.; Morrell, A.P.; Addison, O.; Wehmeyer, C.; Buckley, C.D.; Cooper, M.S.; Lavery, G.G.; et al. 11ß-HSD1 Plays a Critical Role in Trabecular Bone Loss Associated with Systemic Glucocorticoid Therapy. Arthritis Res. Ther. 2019, 21,1-10. [CrossRef] 
69. Schepper, J.D.; Collins, F.; Rios-Arce, N.D.; Kang, H.J.; Schaefer, L.; Gardinier, J.D.; Raghuvanshi, R.; Quinn, R.A.; Britton, R.; Parameswaran, N.; et al. Involvement of the Gut Microbiota and Barrier Function in Glucocorticoid-Induced Osteoporosis. J. Bone Miner. Res. 2020, 35, 801-820. [CrossRef] [PubMed]

70. Ohlsson, C.; Nilsson, K.H.; Henning, P.; Wu, J.; Gustafsson, K.L.; Poutanen, M.; Lerner, U.H.; Movérare-Skrtic, S. WNT16 Overexpression Partly Protects against Glucocorticoid-Induced Bone Loss. Am. J. Physiol. Endocrinol. Metab. 2018, 314, E597-E604. [CrossRef] [PubMed]

71. Bergström, I.; Isaksson, H.; Koskela, A.; Tuukkanen, J.; Ohlsson, C.; Andersson, G.; Windahl, S.H. Prednisolone Treatment Reduces the Osteogenic Effects of Loading in Mice. Bone 2018, 112, 10-18. [CrossRef] [PubMed]

72. Dubrovsky, A.M.; Nyman, J.S.; Uppuganti, S.; Chmiel, K.J.; Kimmel, D.B.; Lane, N.E. Bone Strength/Bone Mass Discrepancy in Glucocorticoid-Treated Adult Mice. JBMR Plus 2021, 5, e10443. [CrossRef]

73. Adhikary, S.; Kothari, P.; Choudhary, D.; Tripathi, A.K.; Trivedi, R. Glucocorticoid Aggravates Bone Micro-Architecture Deterioration and Skeletal Muscle Atrophy in Mice Fed on High-Fat Diet. Steroids 2019, 149, 108416. [CrossRef] [PubMed]

74. Alam, I.; Oakes, D.K.; Reilly, A.M.; Billingsley, C.; Sbeta, S.; Gerard-O’Riley, R.L.; Acton, D.; Sato, A.; Bellido, T.; Econs, M.J. Overexpression of WNT16 Does Not Prevent Cortical Bone Loss Due to Glucocorticoid Treatment in Mice. JBMR Plus 2019, 3, e10084. [CrossRef] [PubMed]

75. Mohan, G.; Lay, E.Y.A.; Berka, H.; Ringwood, L.; Kot, A.; Chen, H.; Yao, W.; Lane, N.E. A Novel Hybrid Compound LLP2A-Ale Both Prevented and Rescued the Osteoporotic Phenotype in a Mouse Model of Glucocorticoid-Induced Osteoporosis. Calcif. Tissue Int. 2017, 100, 67-79. [CrossRef] [PubMed]

76. Wang, F.S.; Lian, W.S.; Weng, W.T.; Sun, Y.C.; Ke, H.J.; Chen, Y.S.; Ko, J.Y. Neuropeptide Y Mediates Glucocorticoid-Induced Osteoporosis and Marrow Adiposity in Mice. Osteoporos. Int. 2016, 27, 2777-2789. [CrossRef] [PubMed]

77. Marenzana, M.; Greenslade, K.; Eddleston, A.; Okoye, R.; Marshall, D.; Moore, A.; Robinson, M.K. Sclerostin Antibody Treatment Enhances Bone Strength but Does Not Prevent Growth Retardation in Young Mice Treated with Dexamethasone. Arthritis Rheum. 2011, 63, 2385-2395. [CrossRef] [PubMed]

78. Henneicke, H.; Herrmann, M.; Kalak, R.; Brennan-Speranza, T.C.; Heinevetter, U.; Bertollo, N.; Day, R.E.; Huscher, D.; Buttgereit, F.; Dunstan, C.R.; et al. Corticosterone Selectively Targets Endo-Cortical Surfaces by an Osteoblast-Dependent Mechanism. Bone 2011, 49, 733-742. [CrossRef]

79. Thompson, D.D.; Simmons, H.A.; Pirie, C.M.; Ke, H.Z. FDA Guidelines and Animal Models for Osteoporosis. Bone 1995, 17, S125-S133. [CrossRef]

80. Yao, W.; Cheng, Z.; Busse, C.; Pham, A.; Nakamura, M.C.; Lane, N.E. Glucocorticoid Excess in Mice Results in Early Activation of Osteoclastogenesis and Adipogenesis and Prolonged Suppression of Osteogenesis: A Longitudinal Study of Gene Expression in Bone Tissue from Glucocorticoid- Treated Mice. Arthritis Rheum. 2008, 58, 1674-1686. [CrossRef]

81. Dias, I.R.; Camassa, J.A.; Bordelo, J.A.; Babo, P.S.; Viegas, C.A.; Dourado, N.; Reis, R.L.; Gomes, M.E. Preclinical and Translational Studies in Small Ruminants (Sheep and Goat) as Models for Osteoporosis Research. Curr. Osteoporos. Rep. 2018, 16, 182-197. [CrossRef]

82. Ding, M.; Cheng, L.; Bollen, P.; Schwarz, P.; Overgaard, S. Glucocorticoid Induced Osteopenia in Cancellous Bone of Sheep: Validation of Large Animal Model for Spine Fusion and Biomaterial Research. Spine 2010, 35, 363-370. [CrossRef] [PubMed]

83. Permuy, M.; López-Peña, M.; Muñoz, F.; González-Cantalapiedra, A. Rabbit as Model for Osteoporosis Research. J. Bone Miner. Metab. 2019, 37, 573-583. [CrossRef] [PubMed]

84. Harrison, K.D.; Hiebert, B.D.; Panahifar, A.; Andronowski, J.M.; Ashique, A.M.; King, G.A.; Arnason, T.; Swekla, K.J.; Pivonka, P.; Cooper, D.M.L. Cortical Bone Porosity in Rabbit Models of Osteoporosis. J. Bone Miner. Res. 2020, 35, 2211-2228. [CrossRef] [PubMed]

85. E Langhoff, J.L. Relative Immunosuppressive Potency of Various Corticosteroids Measured in Vitro. Eur. J. Clin. Pharmacol. 1983, 25, 459-462. [CrossRef] [PubMed]

86. Jacobs, J.W.G.; Bijlsma, J.W.J. Glucocorticoids in Rheumatology: Indications and Routes of Administration. Clin. Exp. Rheumatol. 2011, 2, 127.

87. Herrmann, M.; Henneicke, H.; Street, J.; Modzelewski, J.; Kalak, R.; Buttgereit, F.; Dunstan, C.R.; Zhou, H.; Seibel, M.J. The Challenge of Continuous Exogenous Glucocorticoid Administration in Mice. Steroids 2009, 74, 245-249. [CrossRef]

88. Arantes-Rodrigues, R.; Henriques, A.; Pinto-Leite, R.; Faustino-Rocha, A.; Pinho-Oliveira, J.; Teixeira-Guedes, C.; Seixas, F.; Gama, A.; Colaço, B.; Colaço, A.; et al. The Effects of Repeated Oral Gavage on the Health of Male CD-1 Mice. Lab Anim. 2012, 41, 129-134. [CrossRef]

89. Wood, C.L.; Soucek, O.; Wong, S.C.; Zaman, F.; Farquharson, C.; Savendahl, L.; Ahmed, S.F. Animal Models to Explore the Effects of Glucocorticoids on Skeletal Growth and Structure. J. Endocrinol. 2018, 236, R69-R91. [CrossRef]

90. Sengupta, P. The Laboratory Rat: Relating Its Age With Human's. Int. J. Prev. Med. 2013, 4, 624.

91. Andreollo, N.A.; Santos, E.F.; Araújo, M.R.; Lopes, L.R. Rat's Age versus Human's Age: What Is the Relationship? Arq. Bras. De Cir. Dig. ABCD Braz. Arch. Dig. Surg. 2012, 25, 49-51. [CrossRef]

92. Weinstein, R.S.; O’Brien, C.A.; Almeida, M.; Zhao, H.; Roberson, P.K.; Jilka, R.L.; Manolagas, S.C. Osteoprotegerin Prevents Glucocorticoid-Induced Osteocyte Apoptosis in Mice. Endocrinology 2011, 152, 3323-3331. [CrossRef]

93. Plotkin, L.I.; Bivi, N.; Bellido, T. A Bisphosphonate That Does Not Affect Osteoclasts Prevents Osteoblast and Osteocyte Apoptosis and the Loss of Bone Strength Induced by Glucocorticoids in Mice. Bone 2011, 49, 122-127. [CrossRef] 
94. Horton, J.A.; Murray, G.M.; Spadaro, J.A.; Margulies, B.S.; Allen, M.J.; Damron, T.A. Precision and Accuracy of DXA and PQCT for Densitometry of the Rat Femur. J. Clin. Densitom. Off. J. Int. Soc. Clin. Densitom. 2003, 6, 381-390. [CrossRef]

95. Johnson, L.C.; Johnson, R.W.; Munoz, S.A.; Mundy, G.R.; Peterson, T.E.; Sterling, J.A. Longitudinal Live Animal Micro-CT Allows for Quantitative Analysis of Tumor-Induced Bone Destruction. Bone 2011, 48, 141-151. [CrossRef]

96. Becker, K.; Schwarz, F.; Rauch, N.J.; Khalaph, S.; Mihatovic, I.; Drescher, D. Can Implants Move in Bone? A Longitudinal in Vivo Micro-CT Analysis of Implants under Constant Forces in Rat Vertebrae. Clin. Oral Implant. Res. 2019, 30, 1179-1189. [CrossRef] [PubMed]

97. Abadie, E.C.; Devogealer, J.P.; Ringe, J.D.; Ethgen, D.J.; Bouvenot, G.M.; Kreutz, G.; Laslop, A.; Orloff, J.J.; Vanderauwera, P.M.; Delmas, P.D.; et al. Recommendations for the Registration of Agents to Be Used in the Prevention and Treatment of Glucocorticoid-Induced Osteoporosis: Updated Recommendations from the Group for the Respect of Ethics and Excellence in Science. Semin. Arthritis Rheum. 2005, 35, 1-4. [CrossRef]

98. Compston, J.; Reid, D.M.; Boisdron, J.; Brandi, M.-L.; Burlet, N.; Cahall, D.; Delmas, P.D.; Dere, W.; Devogelaer, J.-P.; Fitzpatrick, L.A.; et al. Recommendations for the Registration of Agents for Prevention and Treatment of Glucocorticoid-Induced Osteoporosis: An Update from the Group for the Respect of Ethics and Excellence in Science. Osteoporos. Int. 2008, 20, 497-498. [CrossRef]

99. Van Staa, T.P.; Leufkens, H.G.M.; Cooper, C. The Epidemiology of Corticosteroid-Induced Osteoporosis: A Meta-Analysis. Osteoporos. Int. 2002, 13, 777-787. [CrossRef] [PubMed]

100. Perel, P.; Roberts, I.; Sena, E.; Wheble, P.; Briscoe, C.; Sandercock, P.; Macleod, M.; Mignini, L.E.; Jayaram, P.; Khan, K.S. Comparison of Treatment Effects between Animal Experiments and Clinical Trials: Systematic Review. BMJ 2007, 334, 197-200. [CrossRef] [PubMed]

101. Park, S.; Oh, J.; Son, K.Y.; Cho, K.O.; Choi, J. Quantitative Computed Tomographic Assessment of Bone Mineral Density Changes Associated with Administration of Prednisolone or Prednisolone and Alendronate Sodium in Dogs. Am. J. Vet. Res. 2015, 76, 28-34. [CrossRef] [PubMed]

102. Carnovali, M.; Banfi, G.; Mariotti, M. Liquiritigenin Reduces Osteoclast Activity in Zebrafish Model of Glucocorticoid-Induced Osteoporosis. J. Pharmacol. Sci. 2020, 143, 300-306. [CrossRef] [PubMed]

103. Briot, K. Bone and Glucocorticoids. Annales d'Endocrinologie 2018, 79, 115-118. [CrossRef] [PubMed]

104. Adami, G.; Rahn, E.J.; Saag, K.G. Glucocorticoid-Induced Osteoporosis: From Clinical Trials to Clinical Practice. Ther. Adv. Musculoskelet. Dis. 2019, 11, 1759720X19876468. [CrossRef] [PubMed] 